\title{
Thermopower of Gadolinium Nitride
}

\author{
by \\ Max Ingle \\ A thesis submitted in partial fulfillment for the \\ degree of Master of Science \\ in the \\ Faculty of Science \\ School of Chemical and Physical Sciences
}

March 2020 


\section{Declaration of Authorship}

I, Max Ingle, declare that this thesis titled, 'Thermopower of Gadolinium Nitride' and the work presented in it are my own. I confirm that:

- This work was done wholly or mainly while in candidature for a research degree at Victoria University of Wellington.

- Where any part of this thesis has previously been submitted for a degree or any other qualification at Victoria University of Wellington or any other institution, this has been clearly stated.

- Where I have consulted the published work of others, this is always clearly attributed.

- Where I have quoted from the work of others, the source is always given. With the exception of such quotations, this thesis is entirely my own work.

- I have acknowledged all main sources of help.

- Where the thesis is based on work done by myself jointly with others, I have made clear exactly what was done by others and what I have contributed myself.

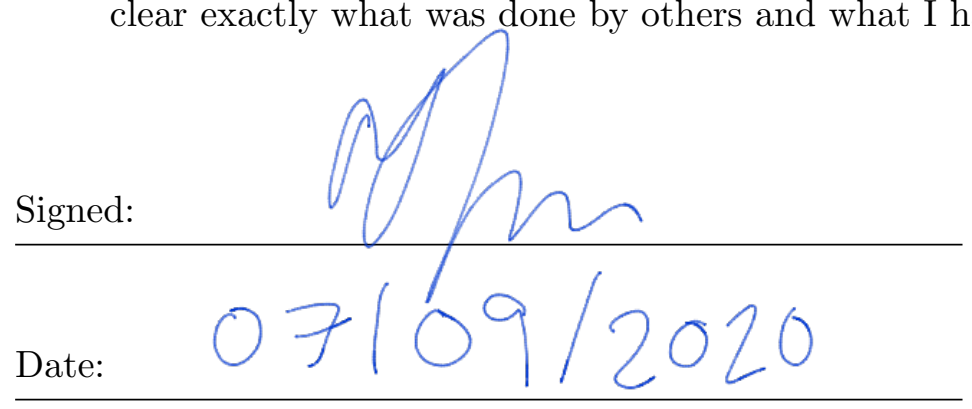


'What the imagination seizes as beauty must be truth.'

John Keats 


\title{
VICTORIA UNIVERSITY OF WELLINGTON
}

\author{
Abstract \\ Faculty of Science \\ School of Chemical and Physical Sciences \\ Master of Science \\ by Max Ingle
}

The following is a report on the thermopower of Gadolinium Nitride. It begins with a discussion of the theory of thermopower. It progresses through simulation, design, and construction of the thermopower measurement stage. It discusses the MBE growth process used to produce the thin-film samples, as well as the subtleties and difficulties associated with making thermopower measurements in situ. It then presents the results of our investigation, and closes with a discussion on possible directions for future work. 


\section{Acknowledgements}

I thank A/Prof. Ben Ruck for his guidance and insight as the principal supervisor of this project.

I also thank Dr. Franck Natali and E/Prof. H. Joe Trodahl for their efforts in directing the Rare-Earth Nitrides group as a whole.

To Dr. Eva Anton, Dr. William Holmes-Hewitt, Jackson Miller, and Ali Shaib, thank you for so willingly sharing your experience and time. 


\section{Contents}

Declaration of Authorship i

Abstract

Acknowledgements

List of Figures vii

1 Introduction $\quad 1$

2 Theory 3

2.1 Diffusion Thermopower . . . . . . . . . . . . . . . . . 3

2.2 Phonon-drag Thermopower . . . . . . . . . . . . . . . 7

2.3 Extra Considerations . . . . . . . . . . . . . . . . . 8

3 Design and Simulation $\quad \mathbf{1 0}$

3.1 Experimental Setup . . . . . . . . . . . . . . . . . . . 10

3.1 .1 The Cryostat . . . . . . . . . . . . . . . . 10

3.1 .2 The Sample Stage . . . . . . . . . . . . . . . . . . . . . . . . . . . . . 12

3.2 The Simulation . . . . . . . . . . . . . . . . . . . 15

4 Sample Growth and Characterisation $\quad 23$

4.1 The UHV Lab . . . . . . . . . . . . . . . . . . . . . . . . 23

5 Thermopower Measurement and Experimental Method 26

5.1 Practical Considerations . . . . . . . . . . . . . . 26

5.2 Mounting the Sample . . . . . . . . . . . . . . . . . . . . . 29

5.3 Cooling the Cryostat . . . . . . . . . . . . . . . . . 30

5.4 Thermopower Measurement . . . . . . . . . . . . . . . . . 31

6 Discussion $\quad 32$

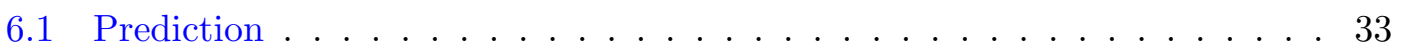

6.2 Results. . . . . . . . . . . . . . . . . . 35

6.3 Error Analysis . . . . . . . . . . . . . . . . . . . 45

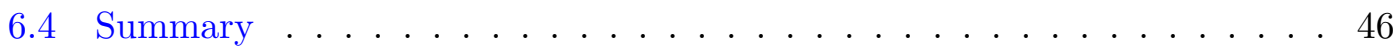


6.5 Future Work . . . . . . . . . . . . . . . . . . . . . . . . . 47

6.5.1 Instrumental Improvements . . . . . . . . . . . . . . . . . . . . . . . . . . . . 47

6.5 .2 Further Investigations $\ldots \ldots$. . . . . . . . . . . 47

$\begin{array}{ll}\text { A Data and Calculations } & 49\end{array}$

$\begin{array}{ll}\text { B References } & 56\end{array}$ 


\section{List of Figures}

3.1 The cryostat $\ldots \ldots \ldots \ldots \ldots \ldots \ldots \ldots$

3.2 Removable stage mount . . . . . . . . . . . . . . . . . . . . . . . . . . . . 12

3.3 Simulation using thermally conductive adhesive . . . . . . . . . . . . . 16

3.4 Simulation using insulating adhesive . . . . . . . . . . . . . 16

3.5 Thermal conductivity of copper vs. temperature . . . . . . . . . . 17

3.6 Thermal conductivity of glass vs. temperature . . . . . . . . . . 18

3.7 Thermal conductivity of teflon vs. temperature . . . . . . . . . . . . 19

3.8 Specific heat capacity of copper vs. temperature . . . . . . . . . . . 20

3.9 Heat capacity of some materials (pyrex for our interest) . . . . . . . . . . 21

3.10 Heat capacity of more materials (teflon for our interest) . . . . . . . . 21

5.1 Thermopower measurement schematic . . . . . . . . . . . . . 26

5.2 Sample stage underside . . . . . . . . . . . . . . . . 28

5.3 Thermopower of our manganin leads . . . . . . . . . . . . . 28

6.1 GdN Thermopower at $\mathrm{T}=58 \mathrm{~K} \ldots \ldots \ldots \ldots$

6.2 GdN Thermopower at $\mathrm{T}=83 \mathrm{~K} \ldots \ldots \ldots \ldots$

6.3 GdN Thermopower at $\mathrm{T}=114 \mathrm{~K} \ldots \ldots \ldots \ldots$

6.4 GdN Thermopower at $\mathrm{T}=140 \mathrm{~K} \ldots \ldots \ldots \ldots$. . . . . . . . . . . . . . . . .

6.5 GdN Thermopower at $\mathrm{T}=164 \mathrm{~K} \ldots \ldots \ldots \ldots$

6.6 GdN Thermopower at $\mathrm{T}=180 \mathrm{~K} \ldots \ldots \ldots \ldots$

6.7 GdN Thermopower at $\mathrm{T}=215 \mathrm{~K} \ldots \ldots \ldots \ldots$

6.8 GdN Thermopower at $\mathrm{T}=250 \mathrm{~K} \ldots \ldots \ldots$

6.9 GdN Thermopower at $\mathrm{T}=280 \mathrm{~K} \ldots \ldots \ldots \ldots$. . . . . . . . . . . . . . . . . . 40

6.10 GdN Thermopower at $\mathrm{T}=308 \mathrm{~K} \ldots \ldots \ldots \ldots$

6.11 GdN Thermopower w/out manganin correction . . . . . . . . . . . . 41

6.12 GdN Thermopower with manganin correction . . . . . . . . . . . . . . 42

6.13 GdN Thermopower with 100*uncertainty . . . . . . . . . . . . . . 42

6.14 GdN Resistivity vs. Temperature . . . . . . . . . . . . . . . . . . . 44

A.1 Thermopower calculations at $\mathrm{T}=58 \mathrm{~K} \ldots \ldots \ldots \ldots$

A.2 Thermopower calculations at $\mathrm{T}=83 \mathrm{~K} \ldots \ldots \ldots \ldots 1$

A.3 Thermopower calculations at $\mathrm{T}=114 \mathrm{~K} \ldots \ldots \ldots \ldots$. . . . . . . 52

A.4 Thermopower calculations at $\mathrm{T}=140 \mathrm{~K} \ldots \ldots \ldots \ldots . \ldots \ldots$

A.5 Thermopower calculations at $\mathrm{T}=164 \mathrm{~K} \ldots \ldots \ldots \ldots$. . . . . . 53

A.6 Thermopower calculations at $\mathrm{T}=180 \mathrm{~K} \ldots \ldots \ldots \ldots$

A.7 Thermopower calculations at $\mathrm{T}=215 \mathrm{~K} \ldots \ldots \ldots \ldots$. . . . . . 54

A.8 Thermopower calculations at $\mathrm{T}=250 \mathrm{~K} \ldots \ldots \ldots \ldots$ 
A.9 Thermopower calculations at $\mathrm{T}=280 \mathrm{~K} \ldots \ldots \ldots \ldots 5$

A.10 Thermopower calculations at $\mathrm{T}=308 \mathrm{~K} \ldots \ldots \ldots \ldots$ 


\section{Chapter 1}

\section{Introduction}

Despite its name, the thermopower of a material is not a measure of an energy transfer rate. Instead, it is a measure of the magnitude of the induced voltage when a temperature difference is maintained across a sample. It is also known as the Seebeck coefficient, named for the Baltic German physicist Thomas Johann Seebeck, and has units of $V \cdot K^{-1}$, although typically measured coefficients are given in units of $\mu V \cdot K^{-1}$. In a general sense, the Seebeck effect is one of three manifestations of thermoelectricity - the other two being the Peltier and the Thomson effects. These effects all fundamentally arise because of the delocalisation of electrons within metals, and as a result the thermoelectric effect is too small to be seen in most materials.

The Seebeck effect occurs when an applied temperature difference causes electrons at the warmer end of the sample to diffuse towards the colder end, causing charge separation and a measurable voltage. The Peltier effect, named for French physicist Jean Charles Athanase Peltier, is its converse and is seen as heat absorption or emission at a thermocouple junction through which a current flows. The Thomson effect, named for William Thomson who would later become known as Lord Kelvin, describes the heating or cooling of a current-carrying conductor with a spatial temperature gradient and a temperature-dependent Seebeck coefficient.

Thermopower and thermoelectric materials are currently being actively researched. There is strong commercial interest in low-mass, high-efficiency heating/cooling devices utilising thermoelectric materials, as well as in new ways to recycle lost heat energy back into usable electricity. The thermoelectric material used most commonly in industry is bismuth telluride $\left(\mathrm{Bi}_{2} \mathrm{Te}_{3}\right)$, but this thesis focuses on testing samples of gadolinium nitride $(\mathrm{GdN})$. 
A gold film sample was used for rough testing of the apparatus. It was made with thermal vapour deposition onto a relatively thin chromium film atop a fused silica wafer. It was selected for two main reasons. The first reason is that gold is atmospherically stable - it does not oxidise or break down during transport or testing. The second reason is that it has a well-known thermoelectric profile - the reliance of the Seebeck coefficient of gold on its temperature is verifiable against other data. This helped us to be sure that there were no obvious errors in the experimental setup prior to commencing tests on more sensitive, expensive, and time-consuming rare-earth nitride samples.

The GdN samples were prepared under ultra-high vacuum (UHV) conditions using molecular-beam epitaxy (MBE). The REN materials in general are a novel class of substance that display both magnetic and semiconductive behaviour over a range of temperatures. They display ferromagnetic behaviour that has been shown to be independent of doping ions, which has strong implications for spintronics research because in principle it enables long-range spin-transport phenomena without impurity-scattering.

Rare-earth nitride materials range from metallic, to semi-metallic, to semiconductive in both their heat- and charge-transport properties, and this thesis investigates the relationship between heat transport and charge transport over a wide range of temperatures. The reader is currently enraptured by Chapter 1 . Chapter 2 discusses the theoretical calculations and their predictions of thermopower measurement phenomena in both general and specific cases. Chapter 3 discusses the experimental simulation, design, and setup. Chapter 4 talks about the methods developed by other scientists in the REN group to grow and characterise the GdN samples. Chapter 5 discusses considerations of, insights into, and difficulties with thermopower measurement under cryostat conditions. Chapter 6 concludes the main body of the thesis by summarising the results gathered thus far. It gives insight into the implications of these results from both a scientific and commercial perspective, and closes with a discussion of future research that may follow. 


\section{Chapter 2}

\section{Theory}

As stated earlier, the term 'thermopower' encompasses three separately measurable effects - the Seebeck, Peltier, and Thomson effects. The primary focus of this thesis is on measuring the Seebeck coefficient of epitaxially grown rare-earth nitride samples. Thermopower data taken across a large temperature range will often show the Seebeck coefficient to be a function of temperature, and the thesis derives, measures, and compares theoretical model calculations with experimental findings. There are two main contributors to the Seebeck effect within a conductor: diffusion thermopower and phonon-drag thermopower.

\subsection{Diffusion Thermopower}

Diffusion thermopower is the thermopower resulting from simple diffusion of hot electrons towards the relatively cool end of the sample. The electrons at the hot end of the sample have a higher average kinetic energy than those electrons at the cool end and thus, on average, they will diffuse across the sample from hot end to cold end. This results in a build-up of electrons at the cooler end of the sample, and this build-up continues until the counteractive electric field is strong enough to prevent electrons from diffusing any further under the fixed temperature gradient. ${ }^{[1]}$ 
As an example, consider an isolated quadrilateral conductor, with an applied temperature gradient. Electron diffusion begins, and the linear motion of such electrons constitute a current whose density $J$ is given by:

$$
J=\sigma\left(-\nabla V+E_{\text {emf }}\right)
$$

where $\sigma$ is the material electrical conductivity, $\nabla V$ is the local voltage, and $E_{\text {emf }}$ is the electromotive force resulting from the charge build-up at the cool end of the sample. Also note that:

$$
E_{e m f}=-S \nabla T
$$

Once a steady state is reached, current density $J=0$, and equations 2.1 and 2.2 combine and simplify to yield:

$$
\nabla V=-S \nabla T
$$

which, after restricting transport phenomena to one dimension only, further simplifies to:

$$
S=\frac{-\Delta V}{\Delta T}
$$


If we compare these electrochemical derivations to a thermodynamic description of the same situation, we find that:

$$
e E \Delta x=C_{e l} \Delta T
$$

where $e$ is the elementary electronic charge, $E$ is the electric field strength, $\Delta x$ is the length of the sample, $C_{e l}$ is the electronic specific heat, and $\Delta T$ is the temperature spatial gradient. Combining equations 2.4 and 2.5 yields:

$$
S=\frac{C_{e l}}{e}
$$

The electronic specific heat capacity $C_{e l}$ is given by: ${ }^{[2]}$

$$
C_{e l}=\frac{\pi^{2} k_{b}^{2} T}{2 \epsilon_{f}}
$$

where $k_{b}$ is Boltzmann's constant, and $\epsilon_{f}$ is the Fermi level of the sample material. Combining equation 2.7 with equation 2.6 yields:

$$
S_{d}=\frac{\pi^{2} k_{b}^{2} T}{2 e \epsilon_{f}}
$$

which describes the thermopower of a material due to electronic diffusion. However, the preceding derivation has assumed that the electronic scattering probability is independent of its temperature. Physically, this means that the electrons moving along the temperature gradient would have the same scattering probabilities as those electrons moving in the opposite direction along the electric potential gradient. However, other work has shown that electron-electron scattering (and impurity scattering in general) is temperature-dependent. ${ }^{[3]}$ 
In the case of a spatial temperature gradient, electrons thermally diffusing from the warm end to the cold end will typically experience less scattering than their counterparts moving from the cold end to the warm end under the influence of the built-up electric field. This serves to modify the measured thermopower relative to the predictions made by the model above. These modifications are typically positive but there are situations where such scattering serves to reduce the diffusion thermopower, and even reverse its sign in certain cases. ${ }^{[4]}$

Much theoretical work has been done on the thermopower of pure metals and simple alloys. Mott and Jones derived a general expression for the diffusion thermopower of a pure metal: ${ }^{[5]}$

$$
S_{d}=\frac{\pi^{2} k_{b}^{2} T}{3 e}\left(\frac{\partial \ln \sigma(\epsilon)}{\partial \epsilon}\right)_{\epsilon=\epsilon_{f}}
$$

where $T$ is the fundamental temperature, and $e$ is electronic charge. Here, $\sigma$ is the electrical conductivity as a function of the Fermi level as a variable $\epsilon$, and $\epsilon_{F}$ is the actual Fermi level of the metal in question.

The above is known as the Mott Formula and while quite general it is subject to a number of constraints. The most prominent of these is that the formula only applies across the range of temperatures for which there are common thermal and electrical relaxation times i.e. for $T>\theta_{D}$ and $T<<\theta_{D}$, where $\theta_{D}$ is the Debye temperature. The other two assumptions made are that $\sigma(\epsilon)$ is smooth and well-behaved as $\epsilon \rightarrow \epsilon_{F}$, and that $k_{B} T<<\epsilon_{F}$.

The astute reader will have realised by this point that none of these assumptions can be reliably applied to novel materials like magnetic semiconductors, nor can they be applied across the wide range of temperatures available to the cryostat user. Thermal and electrical relaxation times typically dominate different temperature regimes. This is due to the complex and dynamic relationship between electrons (driven by thermal and potential gradients) and phonons (driven by thermal gradients only). Electron-electron, electron-phonon, and phonon-phonon scattering processes individually vary from elastic to inelastic depending on both the temperature and the presence/absence of dopants or impurities and serve to mediate a material's electrical and thermal conductivity, as well as its specific heat capacity. This insight leads the reader onto the next section. 


\subsection{Phonon-drag Thermopower}

As indicated earlier in the thesis while discussing the Mott formula, diffusion thermopower varies linearly with temperature, but only over the regions in which there are common thermal and electrical relaxation times. It correctly predicts the relationship between a substance's heat capacity and temperature at very low temperatures, and at high temperatures an approximation can be made which recovers the Dulong-Petit law. ${ }^{[6]}$ It is the intermediate temperature regime, across which one approaches and then recedes from the Debye temperature, that suffers in accuracy.

When making thermopower measurements, we introduce a complication that was not present when these models were being derived - a spatial temperature gradient. Obviously this is necessary for measurement of the Seebeck effect by definition, but it destroys the assumption that scattering processes in a material have radially symmetric dispersion probabilities. Electrons, in general, will be scattered less on their way down the thermal gradient, than they would trying to move back up under the influence of the built-up electric field. Not only that, but these scattering processes themselves are temperature-dependent. When a collision between an electron and another effective mass within the sample (electron, phonon, dopant, e.t.c.), can be considered elastic, the electron leaves the interaction carrying the 'same' amount of energy as it had prior to interaction. However, when such a collision must be considered inelastic, at least some of the electron's kinetic energy is lost as the momentum transfer causes local lattice vibrations instead.

When the sample lattice is in thermal equilibrium, there is no net transport of heat, meaning that there is no net movement of phonons across the sample. When this is the case (at least in a pure crystal), the phonons moving back and forth within the sample form standing nodes which interact with nearby electrons as massive, relatively stationary quasiparticles. Thus, in this case, the charge transport properties of the sample are determined by the degree to which the elasticity/inelasticity of the electron's interaction with the phonon nodes apply.

When applying a thermal gradient for Seebeck coefficient measurements, one is inadvertently causing a net phonon flux from the warm end to the cold end of the sample. The effect that a relatively stationary phonon can have on a passing electron has already been discussed. However, as the temperature falls and the phonon-flux contribution to heat transport grows relative to the electronic contribution, the elastic-interaction assumption becomes weaker and weaker. 
As phonon-flux becomes the primary contributor to heat transport within a temperaturegraded sample, the less-energetic electrons find themselves in a situation where they are dragged along in the wake of such phonons. If a relatively stationary phonon node can deflect a nearby moving electron, it is only logical that a relatively stationary electron would be deflected by a nearby phonon moving past. This typically causes a larger charge build-up than would be expected if electronic diffusion were the only contributor, and is known as phonon-drag thermopower.

\subsection{Extra Considerations}

When one measures thermopower, they are only able to measure the sum of diffusion, phonon-drag, and other effects not yet discussed. It is reasonable to expect a negative thermopower in most cases of simple metals, but a positive thermopower measurement is a signal that there are more electrons with energy $\epsilon<\epsilon_{f}$ diffusing up the thermal gradient in response to the built-up charge than there are electrons with energy $\epsilon>\epsilon_{f}$ diffusing down the thermal gradient. Thus, the measured thermopower is representative of the balance between the electrons above the Fermi energy and the electrons below.

So far our discussion has been limited to metals, but the focus of this thesis is on experimentally determining the Seebeck coefficients of some novel semiconductors developed by others in the REN group at Victoria University of Wellington. More specifically, it is on MBE-grown thin-film samples of gadolinium nitride ( $\mathrm{GdN})$, and samarium nitride $(\mathrm{SmN})$. GdN in particular is exciting because its unique ferromagnetism has much potential regarding externally controllable semiconductors. These implications will be investigated more deeply in future work.

The first question that arises when it comes to the thermopower of semiconductors is the question of whether charge transport is accomplished by electrons or holes $(\mathrm{N}$ or P-type). In the absence of other factors diffusion thermopower should be negative in N-type semiconductors, positive in P-type semiconductors, and zero in intrinsic pure semiconductors. This is simply due to the majority charge carrier moving under the thermal gradient being either positive or negative depending on the dopant. 
The second question is the effect of the band-gap present in semiconductors. Unlike pure metals, the electrical resistivity of a semiconductor falls as its temperature rises due to the presence of an 'energy staircase' that local heat must assist charge carriers to surmount. This means that we expect overall charge transport within the sample to fall as temperature falls, and it also means that the lattice at the warm end of the sample will be more electrically conductive rather than less, though such conductivity is unlikely to vary strongly when we restrict ourselves to a $\Delta 5 \mathrm{~K}$ difference across the sample.

The final question considers the effect of dopants within the semiconductor. Previously in the discussion it was established that thermopower measurements in general are very sensitive to the scattering processes occurring within the sample. Doping a material can have a large effect on the thermopower while leaving the resistivity almost unchanged due to the additional scattering mechanism introduced to the charge carriers. The degree to which the new scattering process affects charge transport is much more sensitive to the atomic structure of the doping ion used, as well as to point defects and lattice distortions introduced by its presence. The presence of a doping ion affects the heat- and charge-transport properties of the material separately, and thus thermopower measurement offers a more subtle and specific probe of ionic structure unavailable to standard four-pole resistance measurements. 


\section{Chapter 3}

\section{Design and Simulation}

\subsection{Experimental Setup}

\subsubsection{The Cryostat}

The process of obtaining thermopower measurements is complex by nature - particularly for the low-temperature regimes we are interested in here. Thermoelectric effects occur at a low level in almost all junctions of dissimilar metals. The goal is to minimise and account for these effects while maximising the measurable thermopower of the sample being tested. To obtain consistent, controllable and (most importantly) repeatable thermopower measurements at low temperatures, we use a cryostat.

This particular cryostat is a bath-style cryostat constructed for the REN research group here at Victoria University. It is constructed of five stainless steel cylinders, getting progressively smaller and nested within one another by size. The space between the first and second shells is the vacuum space - emptied by a turbomolecular pump to provide the main form of insulation between sample and lab environment. Between the second and third shells lies the nitrogen space. This space is repeatedly filled with liquid nitrogen to provide the cryostat's cooling. Once cooling is completed, filling this space again

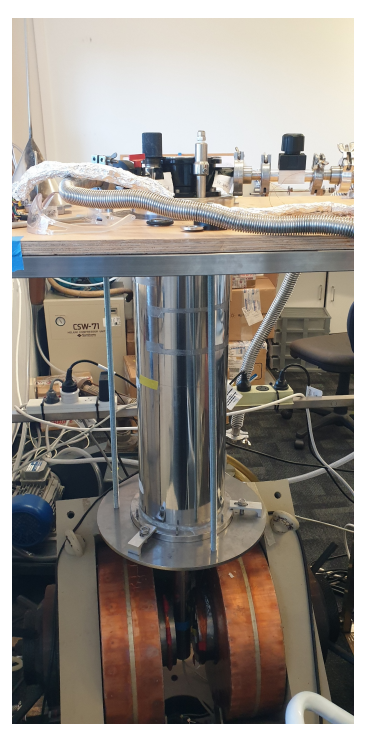

FiguRE 3.1: The cryostat 
allows us to halt warming for the purpose of data acquisition, and helps to maintain temperature stability overnight. The space between the third and fourth shells is another vacuum space. It is common with the first-shell vacuum and emptied simultaneously by the same turbomolecular pump. Between the fourth and fifth shells lies the helium space. This space is filled with liquid helium. This allows sample cooling down to approximately $4.2 \mathrm{~K}$, and using a bellows-style pump on this space can cool the sample further to almost $0.9 \mathrm{~K}$.

The fifth and final cylinder is the sample space containing the sample stage constructed for the purposes of these tests. This space is kept under vacuum separately at all times throughout the experiment. Rogue air currents cooling or warming the temperature sensors are a significant source of noise. They also oxidise and age the rare-earth nitride sample. This piece of apparatus can be removed and placed on a desk mount for sample mounting and repairs.

Unfortunately our cryostat, through years of repeated use, has developed a small leak between the helium space and the common vacuum. This leak does not make itself known to the users until a temperature of approximately $30 \mathrm{~K}$ is achieved, at which point a thermally contracting split opens. After careful analysis and testing it was determined that liquid helium, with its low viscosity and molecular weight, was leaking through this split despite the leak admitting neither air nor liquid nitrogen otherwise. Once the leak begins, the vacuum is lost and heat from the lab environment rapidly soaks in, rapidly boiling off any liquid nitrogen or helium that remains and returning the investigators to step one.

Despite this setback, using liquid nitrogen in both the nitrogen and helium spaces in combination with bellows-pump-mediated evaporative cooling allows us to achieve a stable minimum temperature of $55 \mathrm{~K}$.

The cryostat is vertically mounted and has two removable sample stages. The relatively short and thick thermopower stage reaches the center of the cryostat, but the long and narrow resistivity measurement stage extends a further $45 \mathrm{~cm}$ into the poles of a powerful floor-mounted electromagnet. While we made no use of the magnet for these investigations, theory suggests a strong relationship between an external magnetic field and phonon-thermopower enhancement or attenuation ${ }^{[7,8]}$. Future experiments will attempt to shed light on this relationship. 


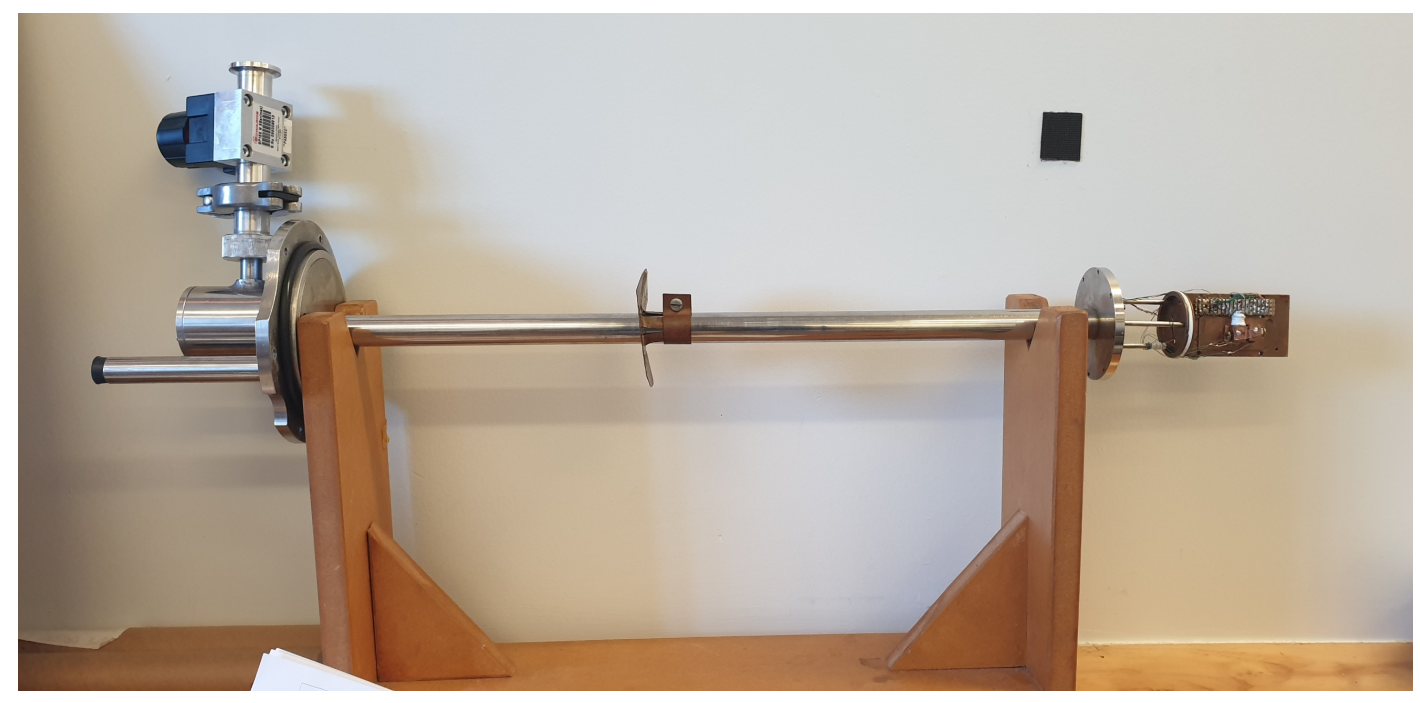

FiguRE 3.2: Removable stage mount

\subsubsection{The Sample Stage}

The sample stage is fitted vertically into the cryostat. As stated above, it is removable to allow sample mounting, maintenance, and repairs. The sample mount itself is constructed of copper, chosen for its high thermal conductivity relative to the stainless steel comprising the bulk of the cryostat.

A number of modular components form the mount:

1. One $1 \mathrm{~cm}^{3}$ copper block

2. One $1 \mathrm{~cm}^{3}$ block, half copper and half Teflon

3. One $26.5 \Omega$ sample heater

4. One $80 \Omega$ stage heater

5. Two LakeShore Cryotronics silicon diode thermometers

The two blocks (copper and copper/Teflon) are mounted parallel to the long axis of the cryostat. The blocks themselves are separated by a $3 \mathrm{~mm}$ gap, with the whole copper 
block set lower than the copper/Teflon combination. The $26.5 \Omega$ sample heater is set into the side of the copper/Teflon block, and the copper/Teflon block is secured to the stage such that the Teflon forms a thermally and electrically insulating barrier between the mounted sample and the rest of the stage.

The two exposed copper surfaces form a flat and parallel region for mounting the sample. The copper and Teflon in combination with the mounted sample heater maintains the thermal gradient needed for making thermopower measurements. The Teflon thermally restricts the heat such that it only flows down through the sample and out through the lower copper block. Each of the two LakeShore Cryotronics silicon diode thermometers are mounted on the copper blocks - one for tracking the warm end of the sample and the other for tracking the cold end. Together, these thermometers provide data on the temperature differential being maintained across the sample.

Wire choice is critical when making any low-temperature electrical measurements. The wires connecting the sample to the equipment in the lab environment also form a directly conductive heat transfer pathway from the lab back down into the sample environment. This is particularly undesirable when making thermopower measurements and properly considered wire choice is just one of many steps taken to mitigate this heat transfer.

Numerous alloys have been developed over the years for low-temperature performance. Our choice in this case is constantan. It is a well-established performer in the field, is widely available, relatively inexpensive, and relatively easy to work with. 
Constantan consists of 55 percent copper and 45 percent nickel. Its main purpose in industry is in strain gauges, where it features due to its low variation in resistivity over a wide range of temperatures. We chose it because its high thermal resistivity relative to more typical wire choices such as copper reduces heat transfer from the lab environment into the sample. Insulated constantan wires of $0.1 \mathrm{~mm}$ diameter were used for all wires not required for powering heaters or thermometers. Insulated copper wires of the same $0.1 \mathrm{~mm}$ diameter carry current to the heaters which are simply coiled constantan. This combination of copper and constantan wires restricts the ohmic heating of the circuit to the heaters only. It lowers the amount of heat transfer into undesirable portions of the sample stage, whilst not requiring excessively high currents to achieve timely warming.

The LakeShore Cryotronics silicon diode thermometers were chosen for their stability and accuracy down to $30 \mathrm{~K}$, and for their almost-linear response to temperature over the whole regime available to the cryostat without liquid helium. They were easily mounted to the sides of each block, and are powered in series by a purpose-built $10 \mu \mathrm{A}$ DC current supply. The wires connecting the $10 \mu \mathrm{A}$ supply with the thermometers are insulated $0.05 \mathrm{~mm}$ diameter copper. Such a low current produces negligible ohmic heating and such a thin wire carries negligible heat into the sample environment. Despite these qualities it proved extremely difficult to work with and suitable alternatives will be investigated for future experiments.

Prior to beginning the experiment, this whole sample stage is covered and sealed by a stainless steel can using a disposable indium wire gasket. The indium wire gasket maintains vacuum viability within the sample space, providing a sterile and still testing environment.

As will be shown later in the thesis, making absolute thermopower measurements in situ is notoriously difficult. In our case the data only provides us with the thermopower of the sample relative to the thermopower of the wires connecting the sample, and so the choice of wires connecting the sample to the nanovoltmeter is critical. Without detailed knowledge of the absolute thermopower of the wires, we cannot calculate the absolute thermopower of the sample. For this we use manganin - another copper/nickel alloy with the addition of manganese. However, it is known that the particular processing methods used in the production of metal wire affects both its mechanical and transport properties $^{[9]}$, and thus two batches of manganin with different origins could have different heat and charge transport properties. In order to achieve absolute thermopower measurement for future rare-earth nitrides, a particular sample of manganin available to the REN group at VUW was carefully tested ${ }^{[10]}(82.2 \% \mathrm{Cu}, 13.3 \% \mathrm{Mn}, 4.5 \% \mathrm{Ni})$, and made available to other investigators on the condition that it was used sparingly. 


\subsection{The Simulation}

Simulations of heat flow within such a piece of apparatus were performed using COMSOL. COMSOL is a powerful, comprehensive, and multifaceted piece of physics modelling software used by scientists and engineers worldwide for computer-assisted-design $(\mathrm{CAD})$ and process optimisation. We used it to help visualise the heat flow and temperature gradients within our stage and sample. It also shed light on how the choice of sample stage adhesive has a strong effect on heat flow rates and temperature gradients within the system.

Due to the delicate nature of the rare-earth nitride sample, temperature sensors cannot be directly mounted to the surface without both damaging it and providing a wayward extra path through which heat can escape. Because of this, the thermometers were fixed to the copper and teflon blocks as near as possible to the ends of the sample. At this point we must make an assumption: that the temperature differential across the blocks as measured by the thermometers is sufficiently similar to the temperature differential maintained across the sample. In this case 'sufficiently similar' means that any discrepancy between block and sample temperature differentials would be equal to or less than the uncertainty of our measuring devices. It is here that the COMSOL simulations demonstrate their value.

The argument is this: if the thermal pathways between the sample and the mounting blocks are more restrictive than the thermal pathway running through the sample, the differential maintained across the sample will be minimal, and the difference between measured block temperatures and inferred sample temperatures will become unacceptably large.

After inspecting the original design notes, and making some measurements with calipers and a micrometer, a CAD file for the sample stage was generated. The stage is constructed of common materials and primitive geometry which made the CAD process relatively simple. Two different simulations were run parallel, one using a poor example of a thermally conductive adhesive (silicone) and the other using a superior example (Ag-based CPU mounting paste). In both cases the adhesive layers were set at $0.01 \mathrm{~mm}$ thick. The sample itself was simulated using an in-built material profile for borosilicate glass which is appropriate given that our rare-earth nitride samples are grown on simple borosilicate microscope slides. 
Presented below is the simulation demonstrating the similarity between measured block temperatures and inferred sample temperatures when a thermally conductive adhesive was used:

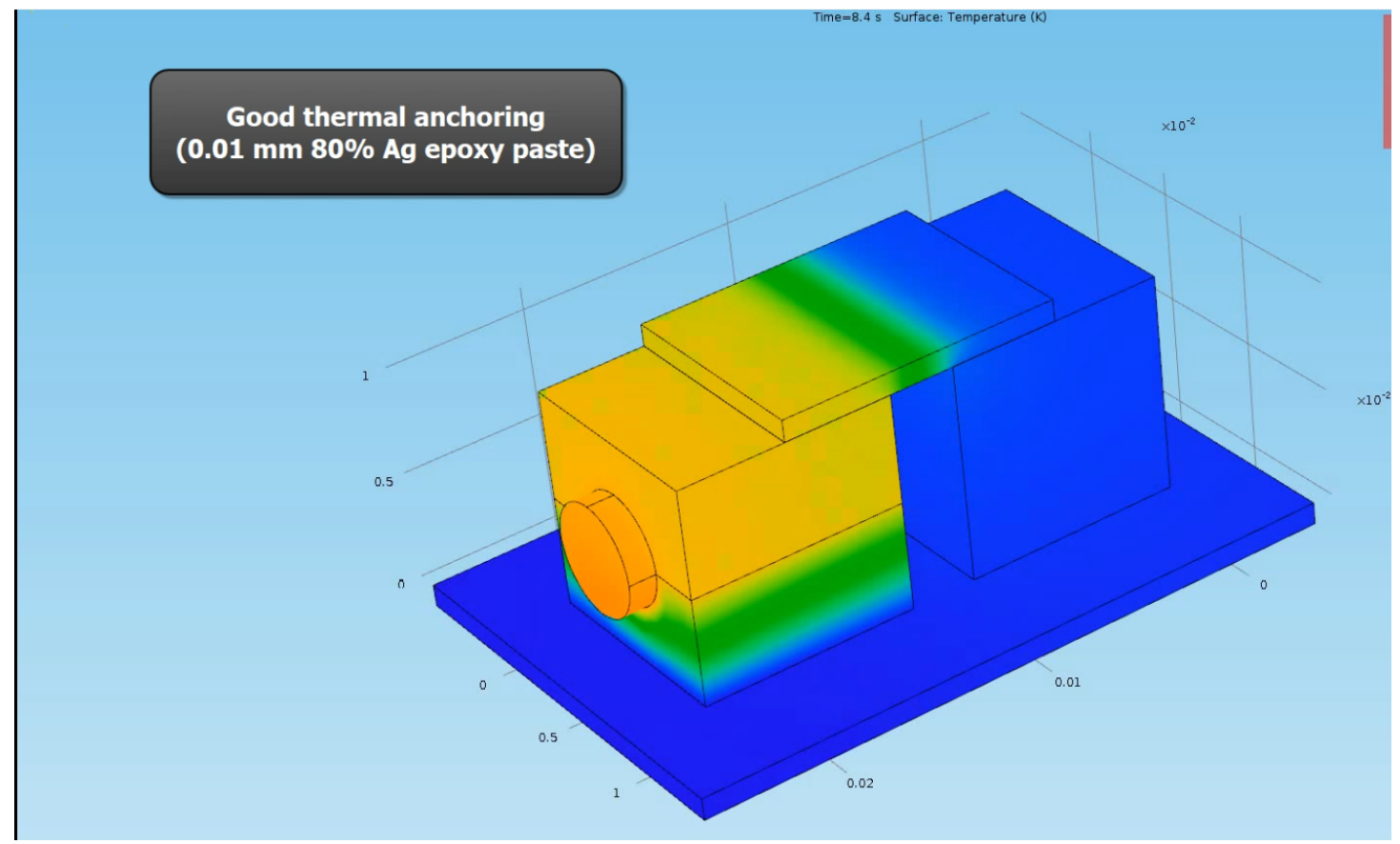

FiguRE 3.3: Simulation using thermally conductive adhesive

Note the agreement between sample and mounting block temperatures as indicated by the colour map of surface temperature in Kelvin. Compare the figure above to the one presented below:

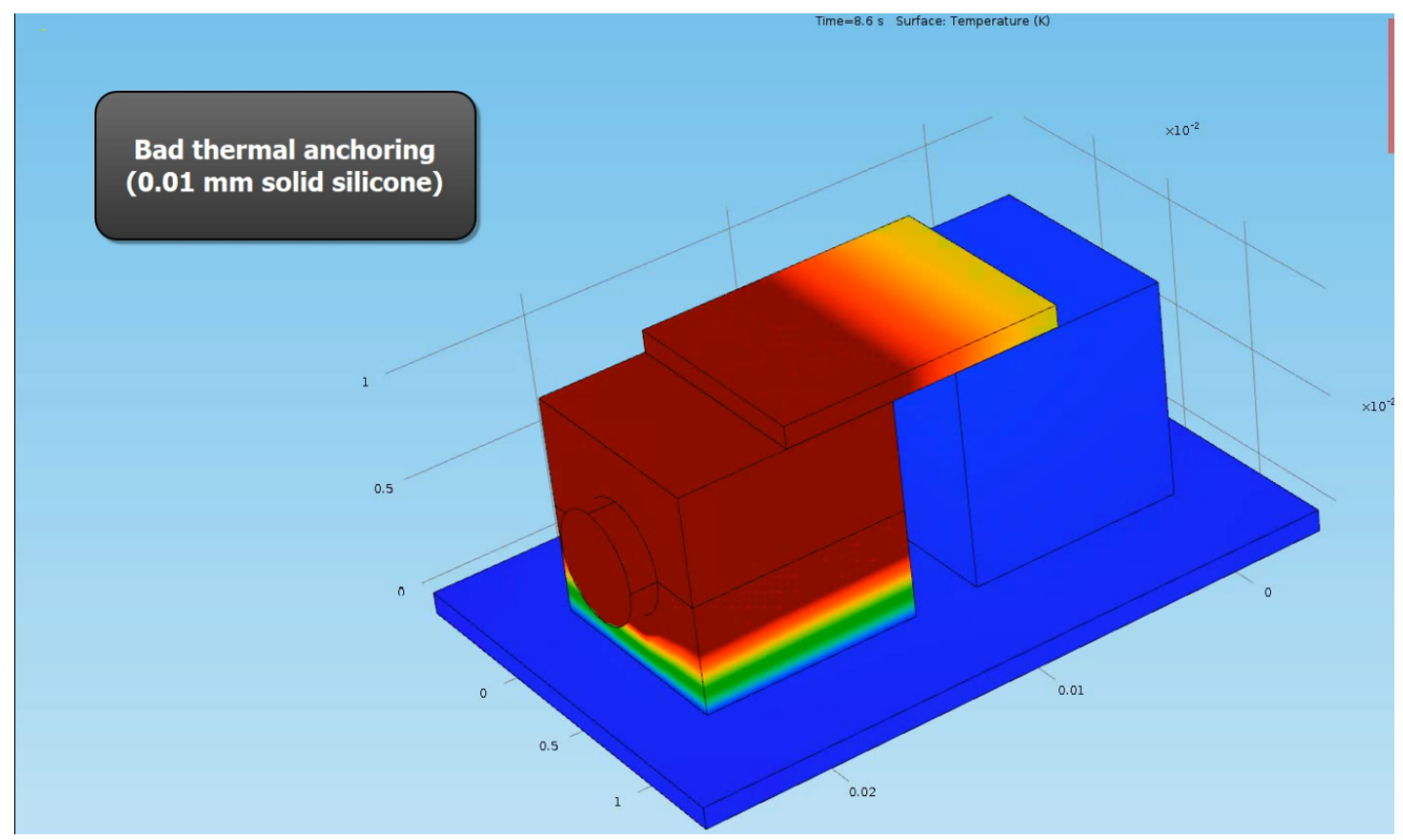

Figure 3.4: Simulation using insulating adhesive 
These simulations are identical except for the change in sample adhesive, and the differences in results are clear. Although not indicated, the colour map of surface temperature in these figures varies from $4 \mathrm{~K}$ to $6 \mathrm{~K}$, and the disagreement between temperatures of the cool end of the sample and the cool mounting block would be comparable to the uncertainty of our thermometers. This would cause an erroneously large $\Delta T$ and our calculated thermopower would be diminished in magnitude.

While simulations such as these have a lot of value, it is also important to note the ways in which they do not capture the subtleties of the physics that apply here. The first major departure from the simulation concerns the thermal conductivity of each of the bulk materials involved (copper, glass, and teflon) ${ }^{[11,12,13]}$.

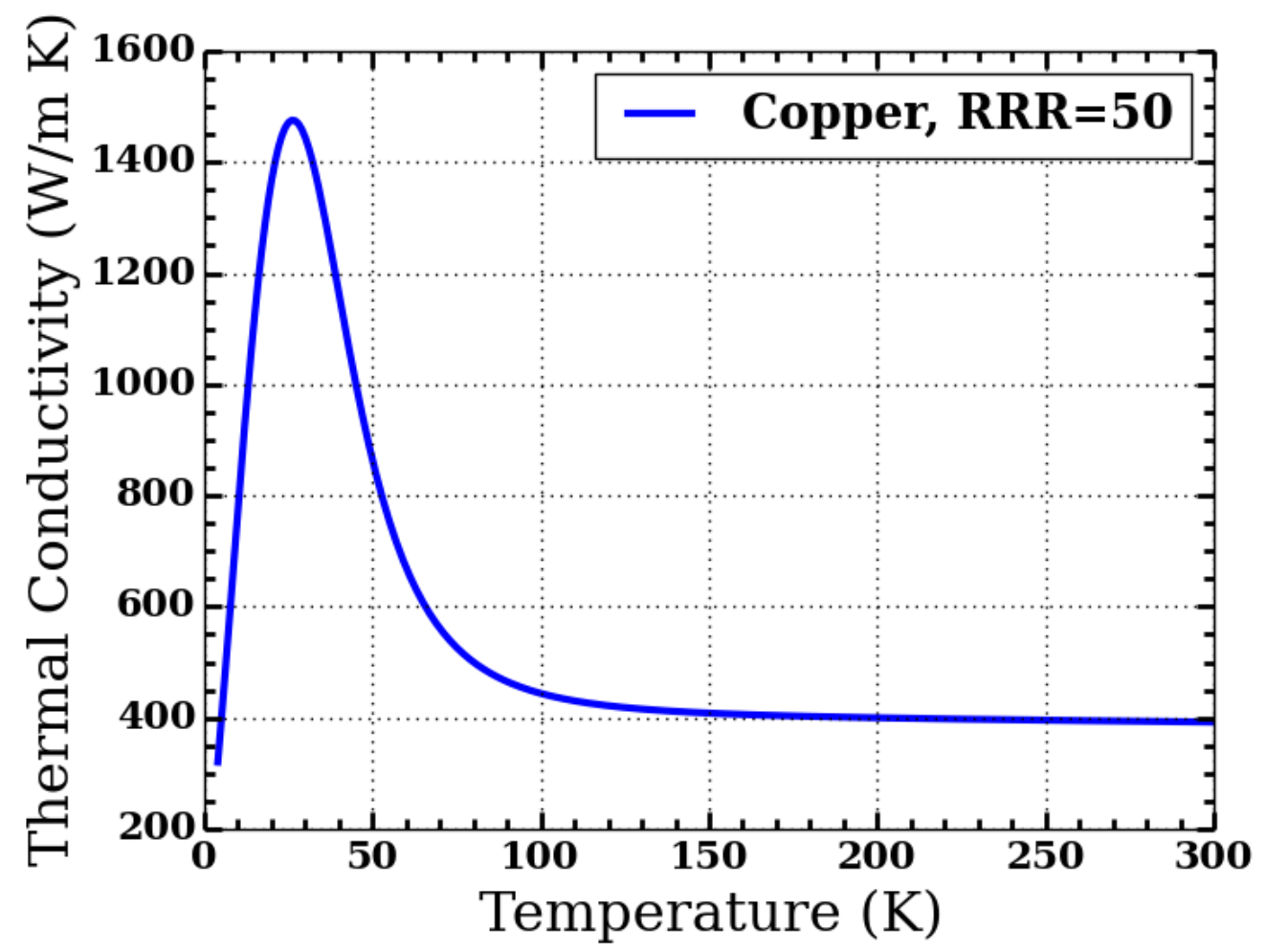

Figure 3.5: Thermal conductivity of copper vs. temperature 


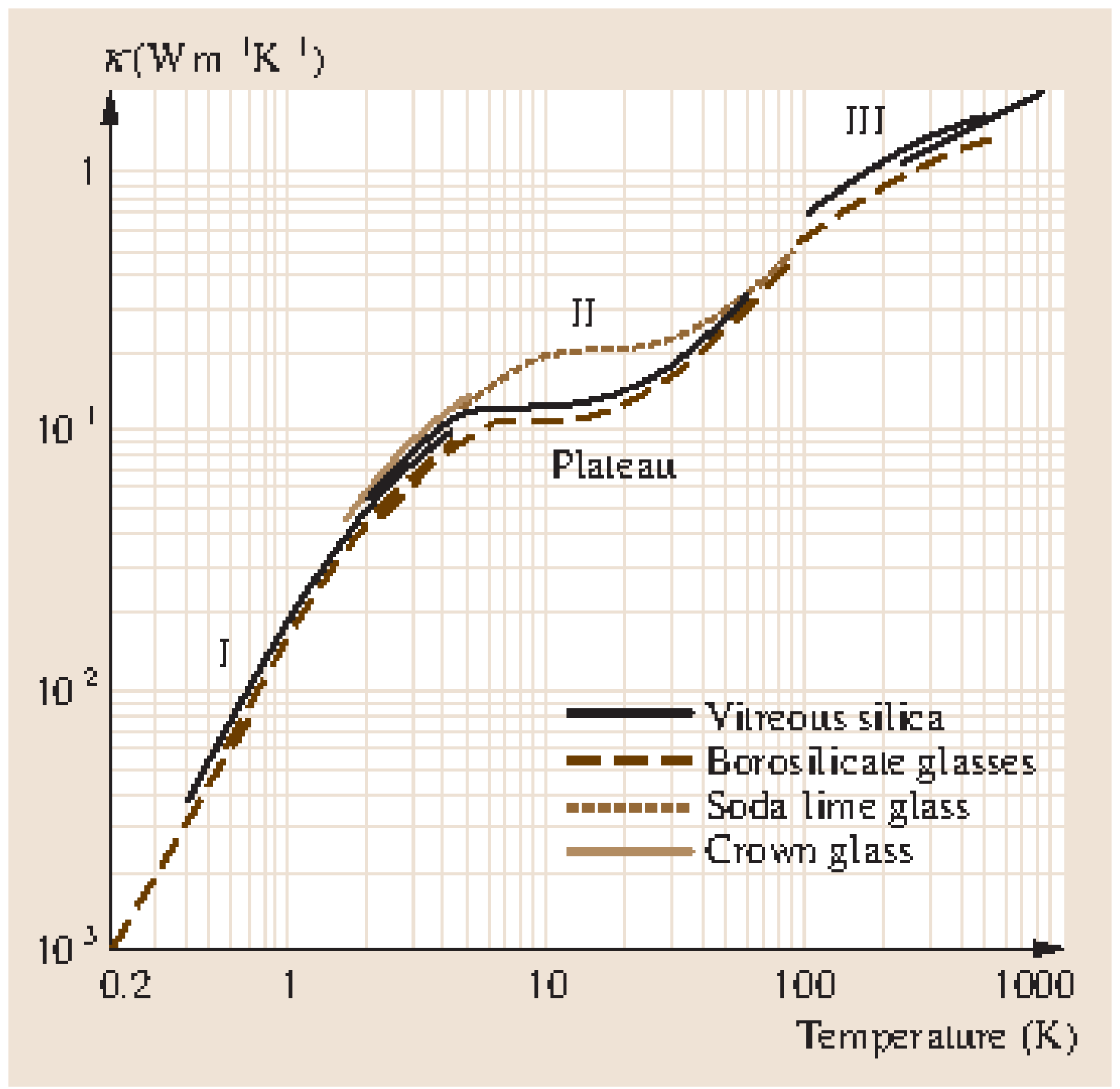

FIGURE 3.6: Thermal conductivity of glass vs. temperature 


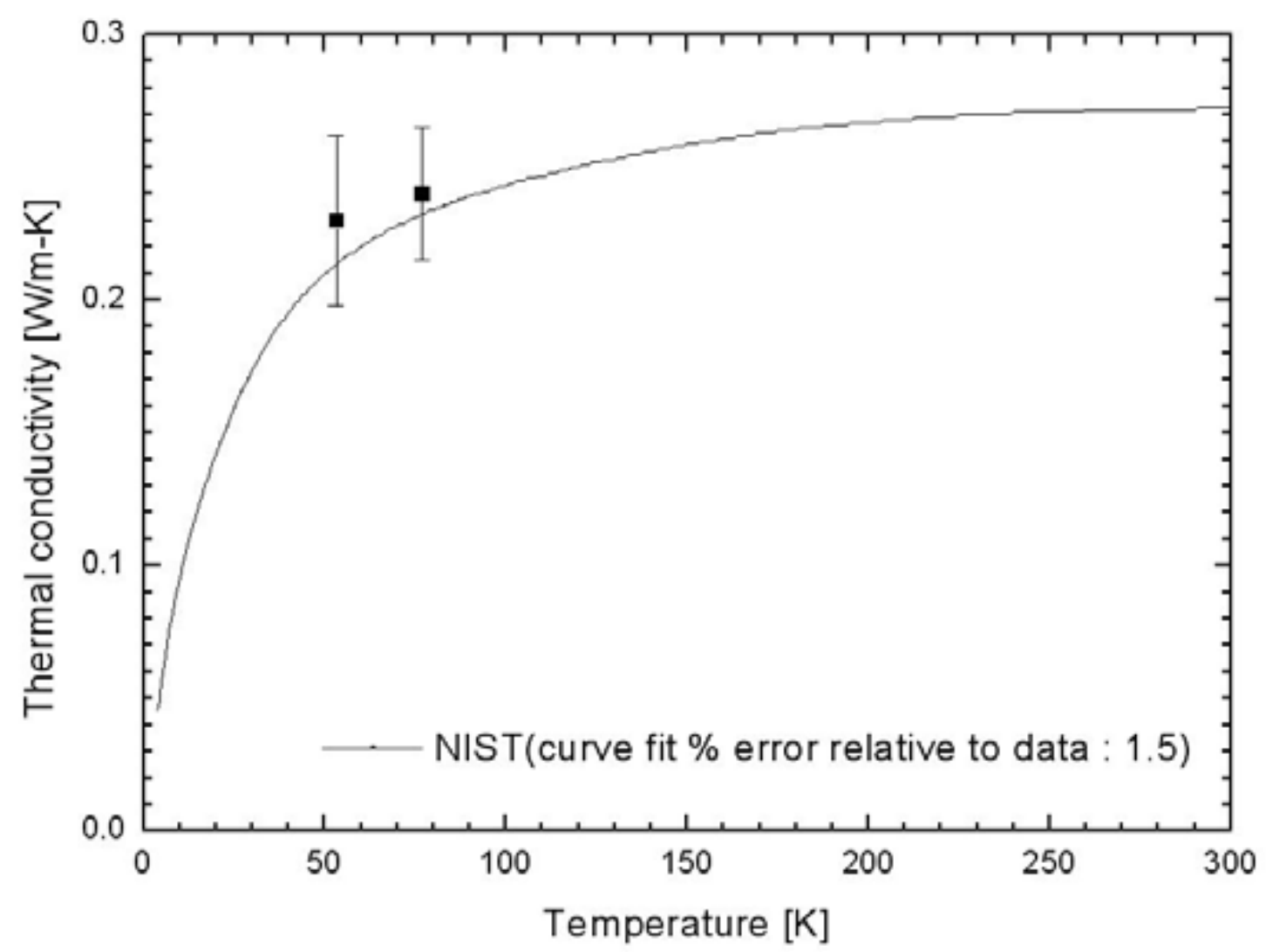

Figure 3.7: Thermal conductivity of teflon vs. temperature 
Teflon's conductivity vs. temperature profile is smooth relative to the profiles of glass and copper. Inadvertently we've avoided the majority of the copper's peak and glass' plateau by using a cryostat that can only reach $55 \mathrm{~K}$. What this meant for the researchers was that both heating power and duration had to be adjusted for the sample stage's temperature. It was quickly noticed that the amount of power necessary for induction and maintenance of $\Delta T$ was much higher at $300 \mathrm{~K}$ than at $55 \mathrm{~K}$.

Compounding these heating power control issues are the variations in specific heat capacity vs. temperature of our materials ${ }^{[14,15,16]}$.

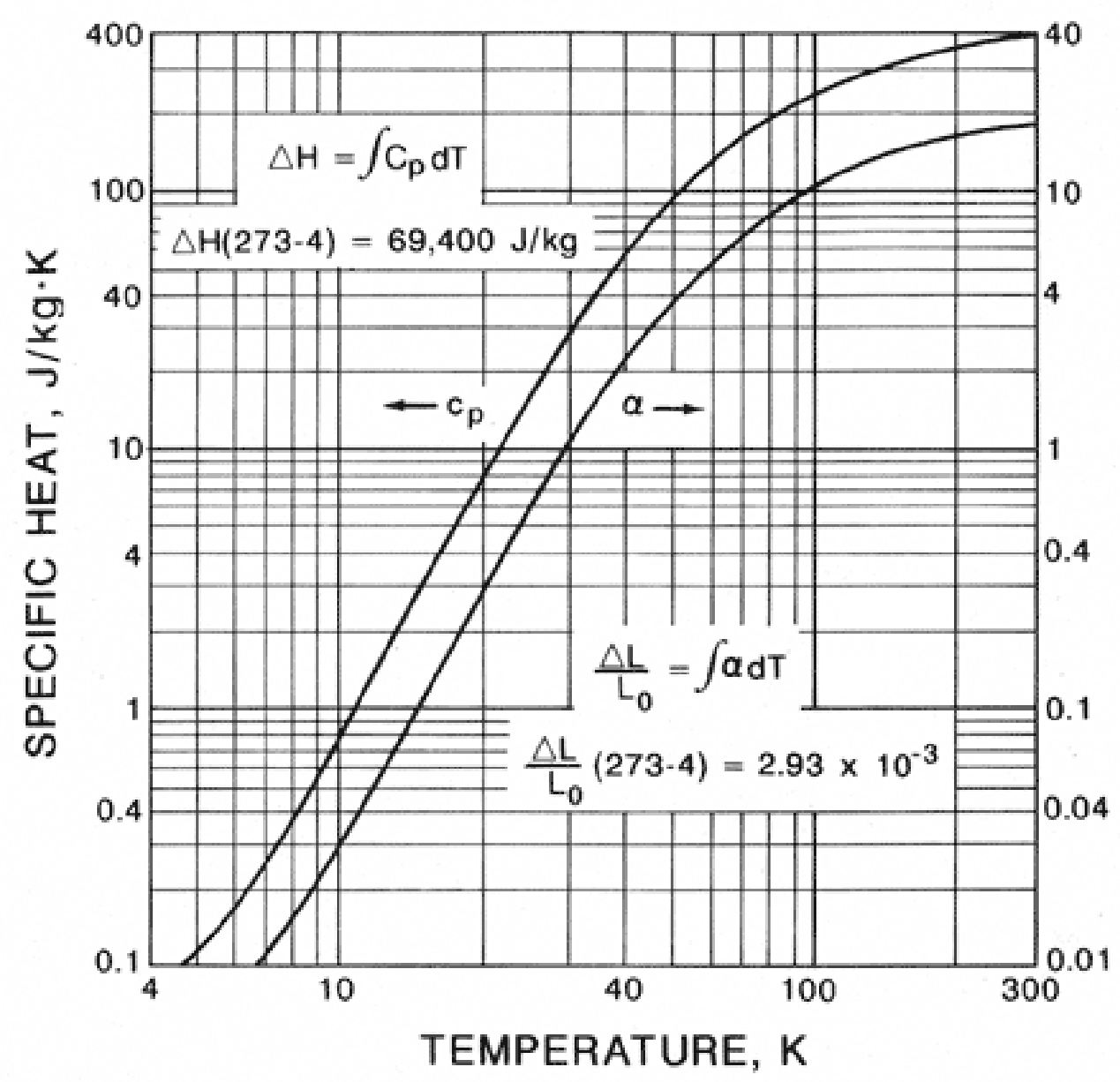

FigURE 3.8: Specific heat capacity of copper vs. temperature 


\section{THERMAL PROPERTIES}

Specific heat capacity curves for some materials

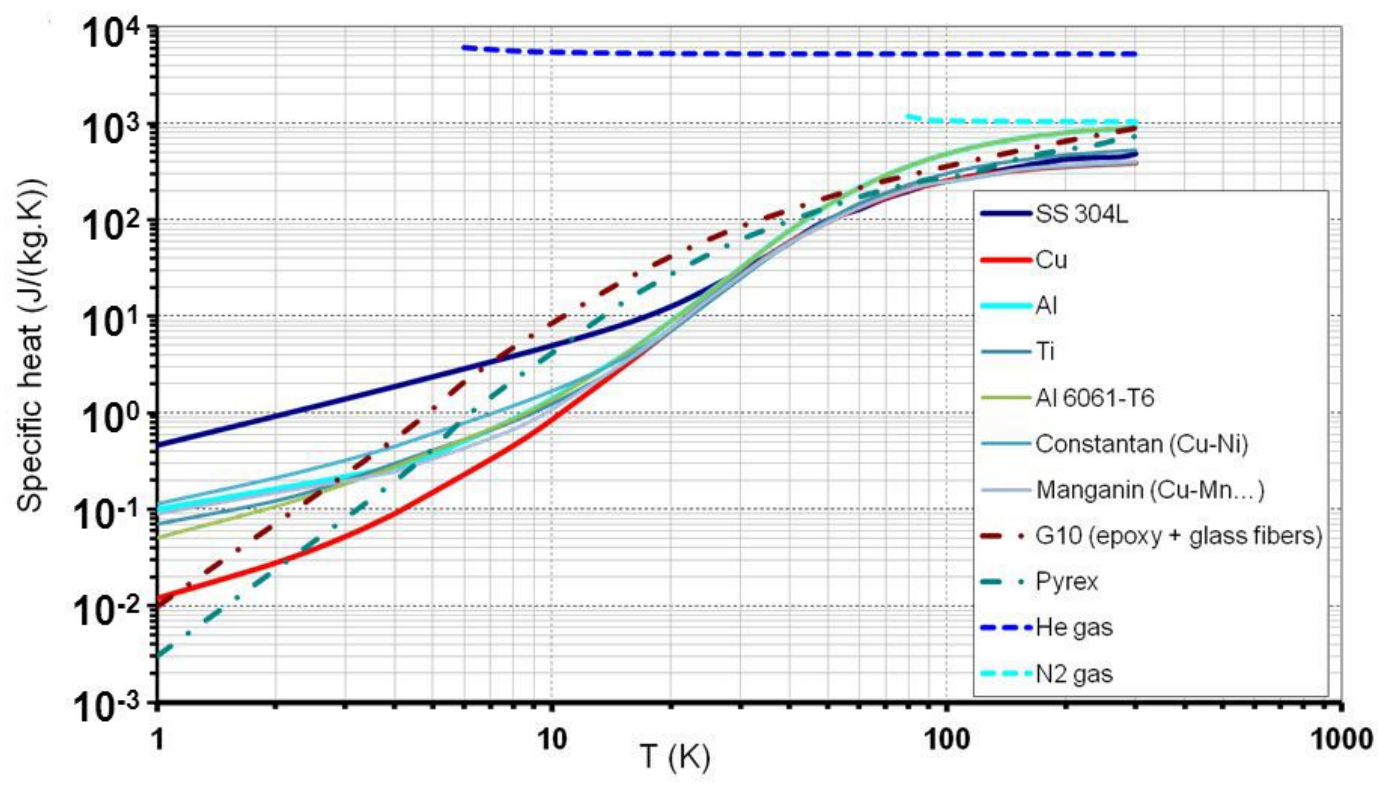

CERN Accelerator School - 2013

Material properties at low temperature

FiguRE 3.9: Heat capacity of some materials (pyrex for our interest)

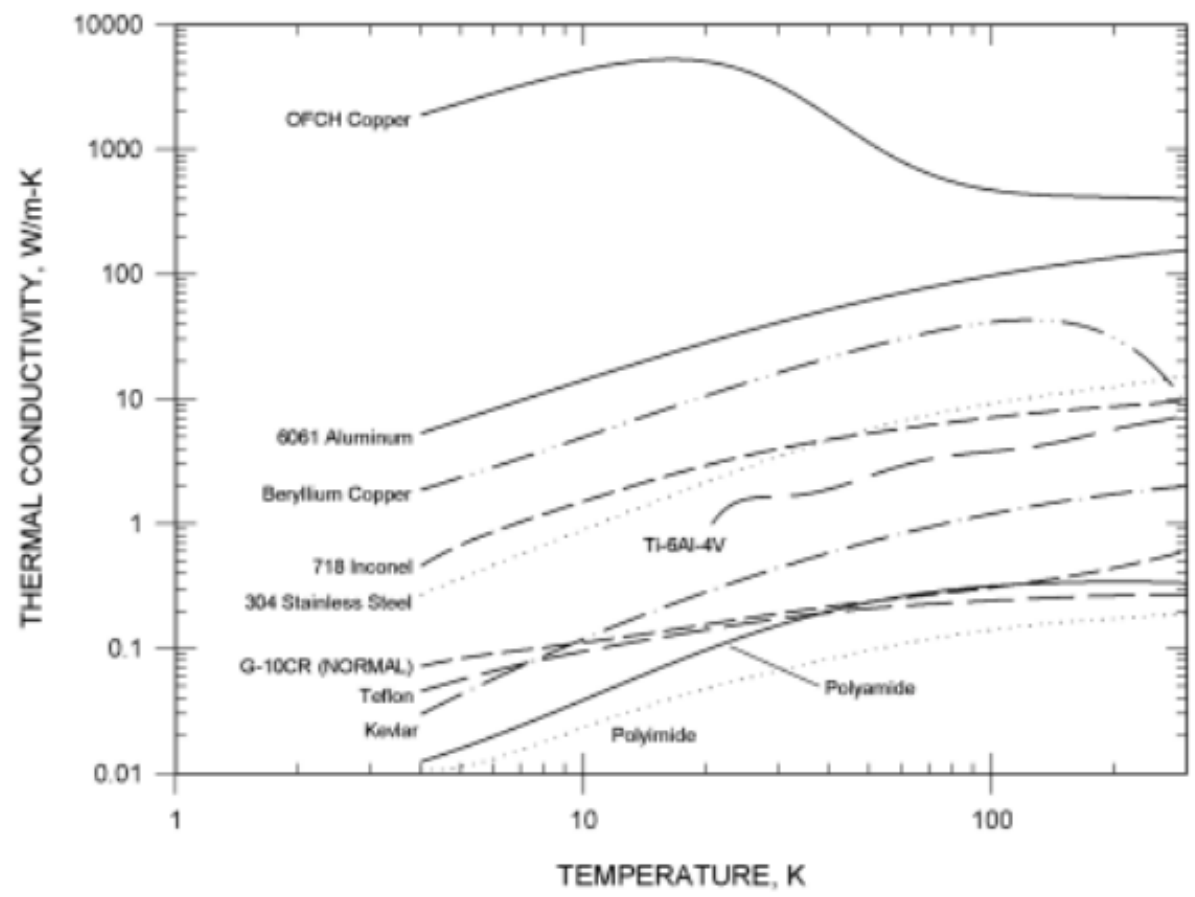

FigURE 3.10: Heat capacity of more materials (teflon for our interest) 
These data show us that the specific heat capacity of our three main construction materials increases non-linearly with temperature over most of the temperature regime that is accessible to us. In practical terms, this means that as the temperature of the system falls, lower power, smaller duration, and greater control of heating current are required for induction and maintenance of an acceptable $\Delta T$. 


\section{Chapter 4}

\section{Sample Growth and Characterisation}

The focus of this thesis is testing the thermopower of two different samples of a rareearth nitride called gadolinium nitride (GdN). The REN group at VUW has put much time and effort into studying the rare-earth mononitride class of semiconductors of which GdN is a member ${ }^{[17]}$. GdN, in particular, is exciting due to its uniquely ferromagnetic behaviour at low temperatures. Attempts to study these materials, up until recently, have been frustrated by fabrication difficulties and the rare-earth mononitride's innate vulnerability to oxidation. However, the advances in ultra-high vacuum (UHV) thin-film deposition technology have made producing and studying these rare-earth mononitrides more accessible to university and private research laboratories.

\subsection{The UHV Lab}

The UHV lab for thin-film deposition is one part of a two-part cleanroom system available to researchers at VUW. Both rooms are sealed behind a pressurised airlock and the device fabrication lab is additionally isolated from all sources of UV radiation. The UHV lab contains two molecular-beam-epitaxy chambers, a low pressure sample storage space, and a nitrogen flowhood work space for preparing bare substrates and fresh samples. 
Samples for the thermopower experiment were prepared in the Thermionics UHV chamber. It is a little older than the Rieber UHV chamber also available, but its use for GdN production is more familiar to the researchers, and it has a quicker turnaround time. It is equipped with resistively heated metal source chambers as well as an electron gun source. The e-gun was used as the energy source for the elemental Gd evaporation. Other metals available for use in the Thermionics system include aluminium and gallium, both of which can be used to form what is known as a passivation layer atop the raw sample. Passivation layers are deposited after growth of the metal nitride of interest because they add a degree of protection from oxygen and moisture attack in the lab environment, however, their insulating nature necessitates pre-sample deposition of gold electrical contacts.

The growth process is as follows:

1. The borosilicate substrate was prepared with gold contacts. Kapton tape was used to mask half of the exposed gold such that the GdN growth would cover half of the substrate longitudinally while leaving bare gold for the indium-tipped manganin connections.

2. The prepared substrate is fixed to the mounting disk and sealed into the loadlock. The load-lock is a secondary chamber that can be vented or evacuated independently of the main chamber. This means that a whole-chamber vent is not necessary each time substrates are loaded or samples are removed.

3. The load-lock is brought down to working pressure $\left(1 \times 10^{-7} \mathrm{mbar}\right)$ using the standard backing/turbopump combination. At this point the load-lock and main chambers are made common, and the substrate is manipulated into position in the center of the chamber directly above the metal sources.

4. After selecting the Gd source, the e-gun is powered up and brought to bear on the metal. The beginning of its sublimation can be noted by the slight but abrupt rise in chamber pressure. Gd vapour attracts other metal gases in the chamber while it travels. Acting as a 'getter', these metal gas complexes subsequently cling to the inner walls of the chamber, and this can be seen as a drop in pressure following the initial rise as sublimation begins.

5. At this point nitrogen gas can be introduced into the chamber. Its flow is strictly controlled by a mass-flow controller and it is split from molecular nitrogen into atomic nitrogen as it enters the chamber. Control of nitrogen pressure during the growth process is what moderates nitrogen-vacancy concentration in the sample. 
These nitrogen vacancies are the dopants in rare-earth mononitride semiconductors. A high nitrogen pressure during growth results in a lower vacancy concentration and a subsequent display of higher electrical resistivity. The converse is also true and it is by varying the nitrogen pressure over multiple growths that we obtain GdN samples with a range of electrical conductivities.

6. Combination of a quartz-crystal detector and a deposition-rate monitor (DRM) (in $\AA \cdot s^{-1}$ ) informs the researchers of the growth rate. Growth time is then adjusted to achieve the desired sample thickness. However, subsequent testing of samples with a Dektak profilometer shows only order-of-magnitude agreement with the DRM, and so the researchers can only be partially sure of the sample thickness. Between $150 \mathrm{~nm}$ and $250 \mathrm{~nm}$ for thermopower samples is desirable. This is slightly thicker than is typical for such thin-film samples, but was done to avoid any z-dimensional electron-scattering suppression.

7. After the desired thickness is achieved, the nitrogen source is closed and the e-gun source is shifted from Gd to either $\mathrm{Al}$ or Ga depending on source availability. The less dense metal sources are depleted more quickly and although aluminium nitride is slightly preferable, gallium nitride is an acceptable alternative when the only other option is to vent the main chamber and refill the $\mathrm{Al}$ source.

8. The process is then repeated with a relatively high nitrogen pressure in order to grow an insulating and oxidation-resistant passivation layer.

9. With the growth process complete, the sources are allowed to cool after which the new samples can be manipulated back into the load-lock chamber. This chamber is then sealed off from the main chamber and allowed to return to lab pressure at which point the fresh samples can be removed and placed into a low-pressure storage system.

Samples are kept at low pressure until all other preparations for their use have been made. Minimising the time that samples spend exposed to ambient pressure and moisture is critical to their preservation and reliable use. Mounting such samples cleanly is a time-sensitive process requiring a steady hand and good familiarity with the measurement apparatus being used. 


\section{Chapter 5}

\section{Thermopower Measurement and Experimental Method}

\subsection{Practical Considerations}

As stated back in Chapter 3, making thermopower measurements in situ is complex. Our measured values correspond to the thermopower of our sample minus the thermopower of the manganin wires.

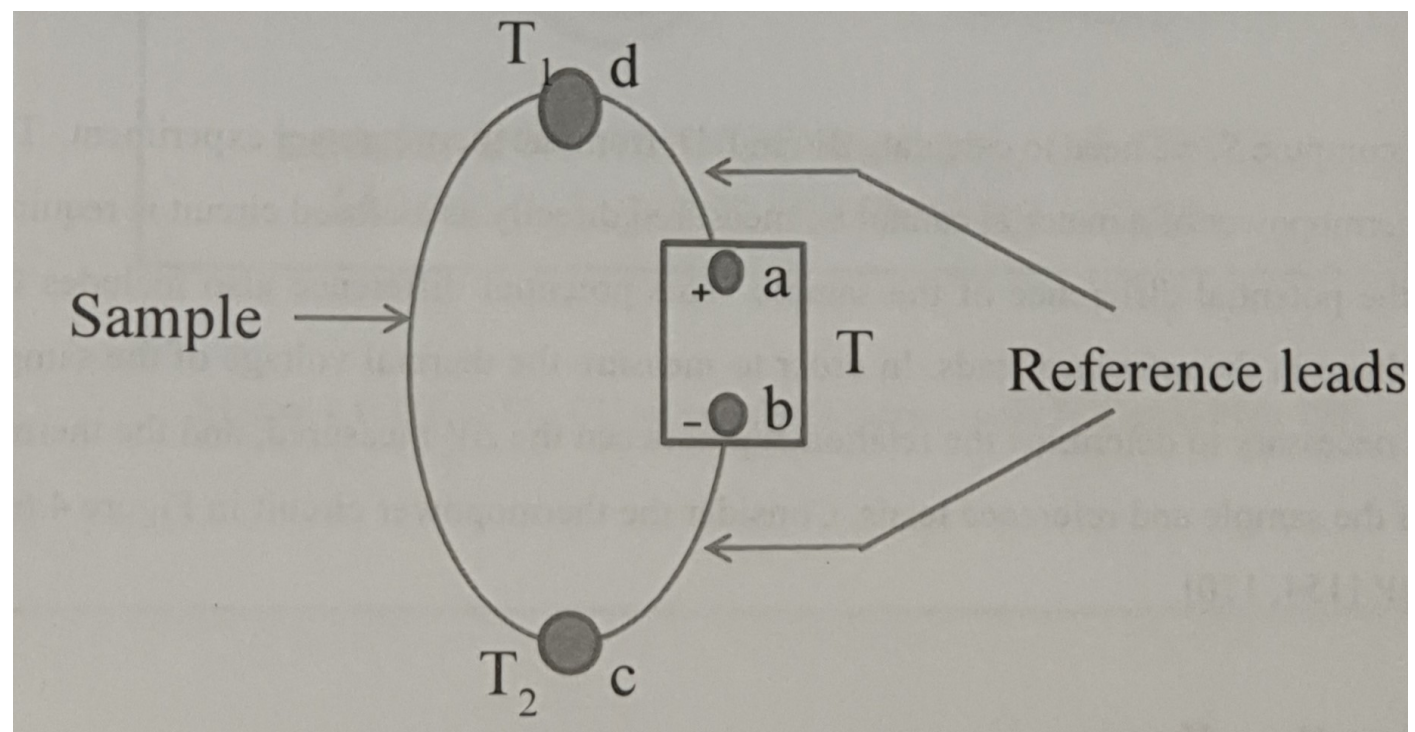

Figure 5.1: Thermopower measurement schematic 
When one measures the voltage across a sample in a situation such as in Fig. 5.1, their multimeter shows $\Delta V=V_{a}-V_{b}$. What is seen is the difference between thermal voltages of the sample and the leads:

$$
\begin{aligned}
\Delta V & =V_{a}-V_{b} \\
& =\left(V_{a}-V_{d}\right)+\left(V_{d}-V_{c}\right)+\left(V_{c}-V_{b}\right) \\
& =\int_{d}^{a} d V+\int_{c}^{d} d V+\int_{b}^{c} d V \\
& =-\int_{d}^{a} S_{\text {leads }} d T-\int_{c}^{d} S_{\text {sample }} d T-\int_{b}^{c} S_{\text {leads }} d T \\
& =-\int_{T_{1}}^{T_{0}} S_{1} d T-\int_{T_{2}}^{T_{1}} S_{s} d T-\int_{T_{0}}^{T_{2}} S_{1} d T \\
& =-\int_{T_{1}}^{T_{2}} S_{1} d T-\int_{T_{2}}^{T_{1}} S_{s} d T \\
& =\int_{T_{1}}^{T_{2}}\left(S_{s}-S_{1}\right) d T
\end{aligned}
$$

Thus it is shown that in order to derive the sample's thermopower vs. temperature profile one must already have the same data profile for the leads connecting the sample to the multimeter. In our case, the leads connecting the multimeter to the sample stage are constantan and the leads connecting the sample surface to the stage are manganin. We have a detailed thermopower vs. temperature profile available for manganin as seen in Ref. 10 and Fig. 5.3, but we have no such information for constantan. To get around this issue, each pair of constantan leads supplying a voltmeter in the lab environment was coiled together and secured to the sample stage frame using teflon tape (see Fig. 5.2). This made sure that any thermal irregularities within the system would cause equal and opposite thermoelectric effects in each pair of constantan leads meaning no net thermoelectric contribution by the constantan. It also helped to sink heat leaking from the lab environment via the connecting leads into the bulk of the apparatus before it affected any sensitive temperature measurements. 


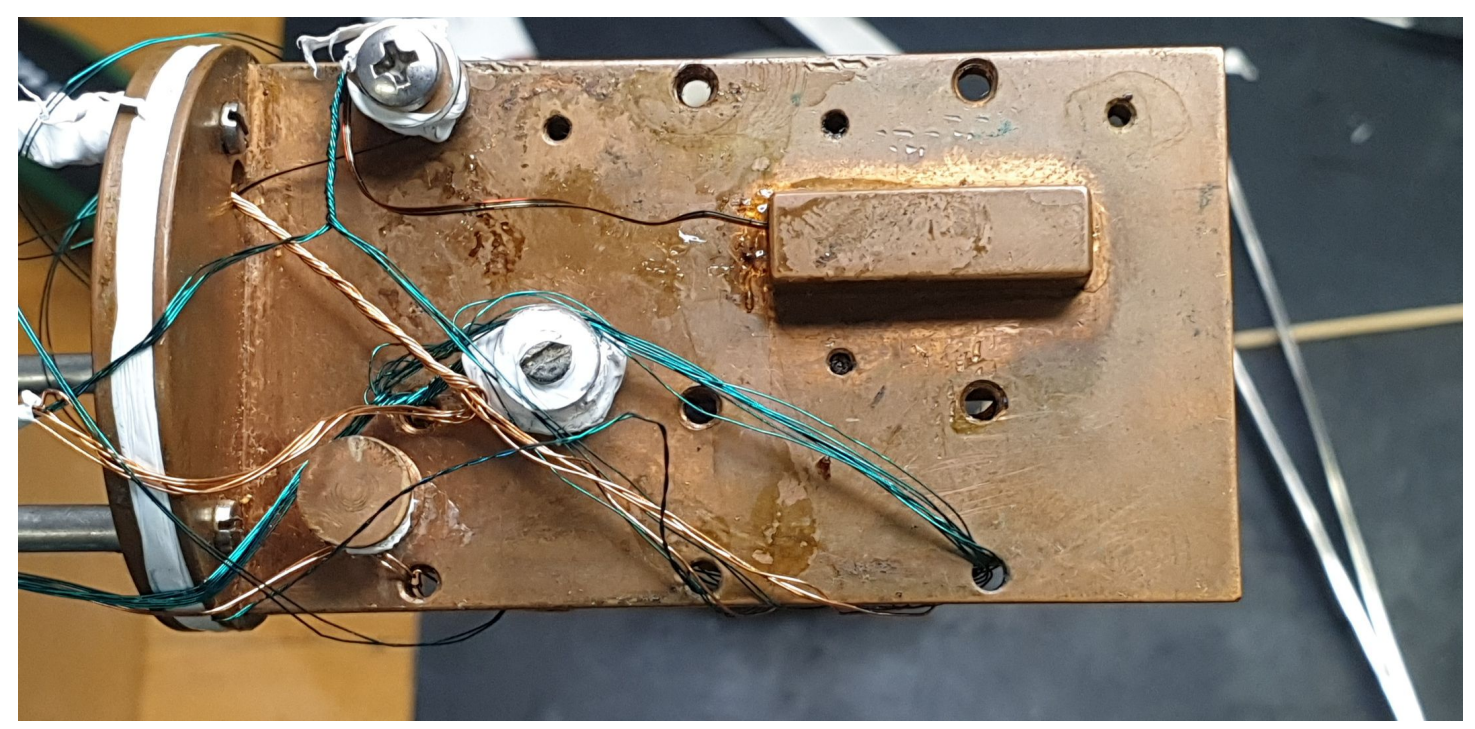

Figure 5.2: Sample stage underside

Thermopower of Manganin

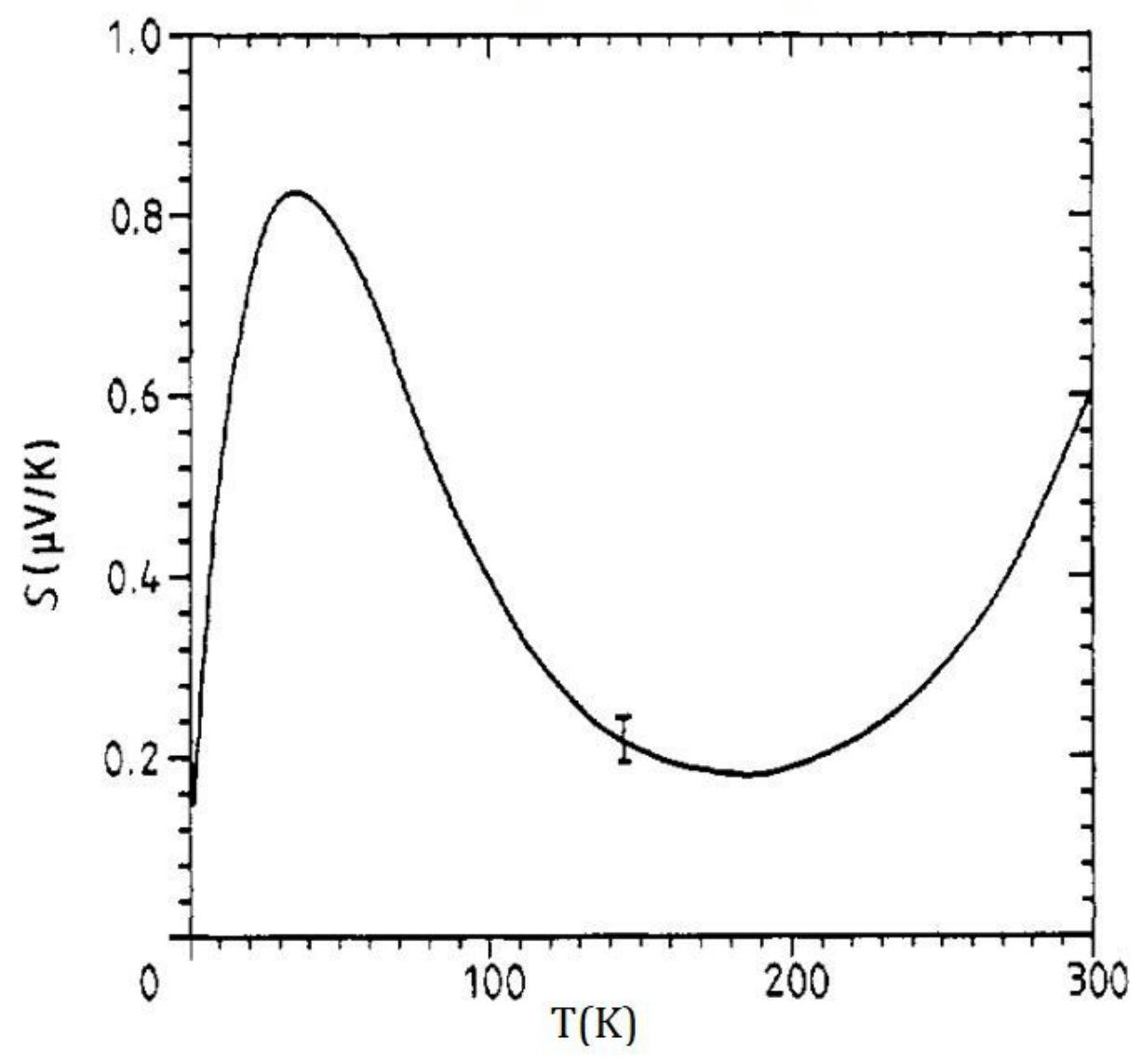

FiguRE 5.3: Thermopower of our manganin leads 


\subsection{Mounting the Sample}

The rare-earth nitride samples prepared by the REN group are sensitive to oxidation and moisture. Once grown in either of the Thermionics or Riber ultra-high-vacuum (UHV) systems, the samples are transferred into a desiccator in the same lab for long-term storage and preservation. Smaller, more portable desiccator chambers are used when the samples need to be transported.

It is critical to minimise the time taken to mount and wire the sample before sealing and evacuating the sample chamber, so some preliminary steps were taken to help the process run quickly and smoothly.

1. A piece of indium wire was lightly greased and cut to appropriate length to form the gasket between the sample stage and the stainless steel vacuum can.

2. Acetone and a microscope lens wipe was used to clean the copper mounting surfaces.

3. The sample contact ends of the manganin wires were de-insulated, cleaned, and tipped with indium contacts. Indium was used because it is highly conductive, and very malleable meaning that it forms reliable electrical connections when squashed tightly against other metals (especially gold). Crucially, it retains these properties at very low temperatures. It can also be prepared before exposing the sample to ambient air, minimising the time spent preparing the sample while it is exposed.

4. The GE varnish used for mounting the thermometers was lightly diluted with toluene prior to sample mounting surface application. The GE varnish available to our lab was rather thick in its native state and while this was acceptable for mounting the thermometers, it was believed that achieving a thin and consistent layer of undiluted varnish between sample and stage would put too much mechanical stress on the sample.

5. The diluted varnish was applied to the copper mounting blocks, spread with a toothpick, and allowed to dry slightly. At this point the desiccator was opened, exposing the sample.

6. Handling the sample only by its edges, it was placed carefully onto the mounting blocks. It was very softly pressed onto the blocks, and smeared back and forth to squeeze out any bubbles in the adhesive varnish beneath.

7. With great care each indium-tipped manganin wire was pressed onto its corresponding exposed gold contact of the sample using the flat side of a screwdriver. 
At this point the prepared indium wire gasket was pressed softly into the thread space in the stainless steel vacuum can, with the ends overlapping slightly to make sure of a complete seal. The can was then bolted onto the mounting plate on the sample stage. The bolts were tightened in opposite pairs to make sure that the indium wire gasket formed evenly. The whole sample stage was then connected to the turbomolecular pump system and evacuated as quickly as possible. The stage was kept horizontal for the first evacuation to make sure that the evaporation of the toluene used to dilute the GE varnish occurred evenly without dislodging or moving the sample. After attaining an internal pressure of roughly $1 \times 10^{-4} \mathrm{~Pa}$ the sample space was sealed off and the pump was powered down and disconnected. The sample stage was lowered into the cryostat and sealed in. The turbomolecular pump system was then connected to the main chamber on the cryostat and powered up.

\subsection{Cooling the Cryostat}

In past experiments it was found that cooling and pumping the cryostat at the same time decreased both cooling and pumping time significantly, but it also increased the rate of liquid nitrogen use to a point that was unsustainable. It also meant that water vapor still in the vacuum space would condense and freeze onto the walls before it could be removed. Upon rewarming of the cryostat this ice sublimates all at once which causes an abrupt pressure change and stresses the turbopump. Thus, pumping was done first and once a vacuum pressure of roughly $1 \times 10^{-5} \mathrm{~Pa}$ was attained, cooling could begin.

The cryostat cannot be completely filled with liquid nitrogen at the beginning of the process. Doing so results in a lot of boiling and spitting which is wasteful and a small but unnecessary risk to the researchers. Instead, a small amount of liquid nitrogen is introduced into the chamber and allowed to settle. As cooling progresses, further introduction of liquid nitrogen becomes less boisterous. At this point, the cooling process is reduced to simply filling the liquid nitrogen space in the cryostat to the top, and leaving it to cool and evaporate. After multiple iterations of the filling and cooling process, the cryostat's internal temperature stabilises at roughly $115 \mathrm{~K}$.

At this point, liquid helium transfer would typically begin. However, as mentioned earlier in the thesis, the cryostat available to the researchers does not sustain a vacuum under liquid helium conditions. Instead, the liquid nitrogen filling and cooling process was repeated for the inner chamber. This put the walls of the removable sample chamber in direct contact with liquid nitrogen, and quickly dropped the temperature to $77 \mathrm{~K}$. 
Once the sample temperature measurements had both stabilised at $77 \mathrm{~K}$ the outer and inner fluid chambers were again filled with liquid nitrogen. The outer chamber was left unsealed to provide evaporative cooling, but the inner chamber was sealed and connected to a bellows-pump. This pump is designed to move large volumes of gas (compare with the turbomolecular pump, designed to pull a hard vacuum). Once the bellows-pump was activated it removed all but the coldest fraction of the liquid nitrogen from within the inner chamber, dropping the sample temperature to $55 \mathrm{~K}$.

\subsection{Thermopower Measurement}

The temperature differential heater was powered with $50 \mathrm{~mW}$ while tracking the temperatures at each end of the sample. While difficult to maintain complete control, the goal was to use the nanovoltmeter to track the thermopower voltage as the temperature differential $\Delta T$ grew from $3 \mathrm{~K}$ to $7 \mathrm{~K}$. Each voltage reading was plotted against its corresponding temperature differential, and the slope of such data yielded the thermopower measurement for the chosen temperature - $55 \mathrm{~K}$ in the first case.

The $\Delta T=5 \mathrm{~K}$ minimum was chosen because readings below this would contribute more uncertainty than useful information. The maximum of $\Delta T=7 \mathrm{~K}$ was chosen because the thermopower was not expected to be linear beyond this point. Such a departure from linearity would skew the derivative calculation method used to process the data.

The method above was repeated for $25 \mathrm{~K}$ intervals as the cryostat warmed. While the stage heater could be employed to speed up the process, it was found that natural warming was sufficient to heat the sample between data acquisition points, at least at first. As the testing temperature rose toward that of the lab environment, a combination of liquid nitrogen cooling and stage heating had to be employed to ensure stability of the sample environment during data acquisition. 


\section{Chapter 6}

\section{Discussion}

The thermopower of pure metals has been well studied over the past 50 years, both theoretically and experimentally. However, the magnitude of the thermopower expressed by pure metals is too small to have much commercial use outside the production of thermocouples. Semiconductors tend to show a stronger thermopower response, but the theoretical treatment of such substances is much more complex than that of pure metals, with modelling and experimentation playing a much more active role in determining the tunable parameters.

Optimisation and commercial production of thermoelectric semiconductors is a highly active area of research, with a particular focus on the engineering of super-efficient thermoelectric heaters, coolers, and recyclers of waste heat. GdN and the unusual ferromagnetism it displays at low temperatures holds much promise for the future of computing. Magnetoresistive random access memory (MRAM), for which GdN is a strong candidate substance, can compute far more data using a given amount of power. Not only is its information density much higher, but the natural ferromagnetism of GdN means that bit-states are retained even after computing power shutdown, so long as cooling is maintained. Thermopower studies of GdN are useful for probing the specifics of charge/heat transport, and their associated scattering processes. There is a future where spatially compact, high-power, low-temperature computers utilising GdN-MRAM are kept cool by super-efficient zero-maintenance thermoelectric refrigerators. Typical random-accessmemory devices require constant refreshing in order to retain the results of calculations performed using them, and the development and commercialisation of low-temperature MRAM devices using GdN would be a significant step forward in materials science, computer science, and engineering. 


\subsection{Prediction}

Electrons contribute significantly to both heat and charge transport within a metallic or semi-metallic substance. These properties are coupled in such a way that it is not a simple case of maximising one relative to the other in order to maximise the Seebeck Effect response.

Physically, the heated electrons are forced to occupy higher energy states. In thermal equilibrium such excited electrons have an equal probability to scatter in any direction the magntitude of these random scattering processes is what constitutes 'temperature' in a bulk substance. However, under an applied temperature differential these heat-excited electrons are more likely to be scattered when moving towards the heat source rather than away from it. Electrons, in their attempt to occupy empty lower energy states, will begin to drift away from the heated end of the sample until they meet electrons already occupying these lower energy states at the cool end of the sample. As the build-up of electrons at the cool end of the sample increases, the charge density begins to repel further incoming electrons. The strength of this electromagnetic repulsion increases until the forces driving electrons from each end of the sample toward the other balance out. When this steady-state is reached the Seebeck coefficient can be measured.

In metals, electrons above and below the conduction band as well as lattice vibrations all contribute to the heat-transporting abilities of the material. However, charge transport is only achieved by electrons already lying in the conduction band. Because of this, electrons within most materials are not transportable by electric or thermal gradients equally. At first glance one would tend to expect the thermopower sign to always match the sign of the charge carrier (negative for metals), but the behaviour becomes more complex as one increases atomic number. Some metals, such as palladium and platinum, show a consistently negative and relatively strong Seebeck coefficient which indicates a high density of states above the conduction band as well as strong scattering of electrons as they attempt to return to the heated end of the sample. In constrast, metals such as copper, silver, and gold show a weaker but consistently positive Seebeck coefficient which indicates a more constrained density of states above the conduction band. Fewer electrons can build up before electrostatic repulsion reverses their drift direction and in these cases the lattice and sub-conduction band contributions to heat transport are enough to dominate the electric potential build-up and drive the majority of electrons against the thermal gradient, resulting in a positive Seebeck coefficient. 
The relative contributions of electrons to charge and heat transport properties of metals vary with temperature. As temperature falls, the electrical conductivity rises meaning that electrons will respond more readily to the built-up electric potential. However, during the same reduction of temperature, the thermal conductivity falls meaning that less heat is required to achieve the same temperature gradient. Ultimately it is this heat that drives the dynamics of the process and so overall we expect suppression of thermopower regardless of sign as the temperature approaches absolute zero.

The electronic, phononic, and lattice contributions to both thermal conductivity and specific heat capacity can be considered separately. Because of the huge difference in effective mass between electrons and phonons, phononic contributions to heat transport are insignificant until lower temperatures begin to suppress electron velocity. Electrons will experience a much greater reduction in velocity (and scattering probability) than phonons over a reduction in temperature and as a result the electronic contribution to both thermal conductivity and specific heat diminishes. Electron-electron scattering dominates at high temperatures, phonon-phonon scattering dominates at near-zero temperatures, but the interaction between electrons and phonons at intermediate temperatures provides the most insight into atomic structure. This knowledge informs us strongly when we turn our attention to semiconductors.

In semiconductors, the differences between heat and charge transport become more subtle. Electrons or holes in semiconductors do not readily carry current until they are energetically promoted to the conduction band either by heat, photons, or some other external source of energy. This means that a semiconductor's ability to carry current $d e-$ creases as its temperature falls, in contrast to pure metals. As a result, as temperatures fall, a greater charge density will be required to force charge carriers back against the applied temperature gradient - increasing thermopower magnitude. Broadly speaking, semiconductors show wide variations in specific heat capacity vs. temperature due to their atomic complexity. Metal alloys, ionic compounds, and doping concentration all affect the degrees of freedom available to electronic and lattice vibrations within the sample. It is because of the decoupling of heat capacity, heat transport, and charge transport properties found within semiconductors that they make such good candidates for tunable thermoelectric properties.

GdN is relatively simple. Its natural atomic arrangement is an insulating rock-salt structure. It has been shown by previous work in the REN group to vary from insulating to metallic behaviour as an increasing number of nitrogen vacancies form the dopants of the substance. As the vacancy concentration increases GdN displays increasingly metallic behaviour. As such it is predicted that GdN will display a minimal thermoelectric 
response at both high and low vacancy concentrations. Low vacancy GdN will not be electrically conductive enough for an applied temperature differential to cause a significant amount of electron drift, and high vacancy GdN will only experience a low charge build-up before reversal of electron drift begins. Thermopower response is predicted to be maximised at a light- to medium-level of doping, sufficient to partially decouple thermal and electric properties whilst retaining reasonable levels of both. Thermopower in metals is typically on the order of $10^{-1} \mu \mathrm{V} \cdot \mathrm{K}^{-1}$. Semiconductors have a much lower inherent electrical conductivity, and this conductivity falls with temperature. It is thus predicted that the thermopower of GdN will be at least an order of magnitude stronger when the optimum nitrogen vacancy concentration is found, and that the most significant deviations from typically metallic behaviour will occur over the temperature regime most associated with the dominance of electron-phonon scattering processes.

\subsection{Results}

Strong thermopower results proved elusive throughout the course of this thesis. The researchers were met with a number of challenges during the investigation including but not limited to; being required to tear down, rebuild, and test the sample stage to make it usable, upskilling in order to use the equipment and modelling software, learning to use the UHV chamber system safely, and navigating experimental research during a nationwide pandemic lockdown. Despite these setbacks, their data on the thermopower of gadolinium nitride is presented below. 


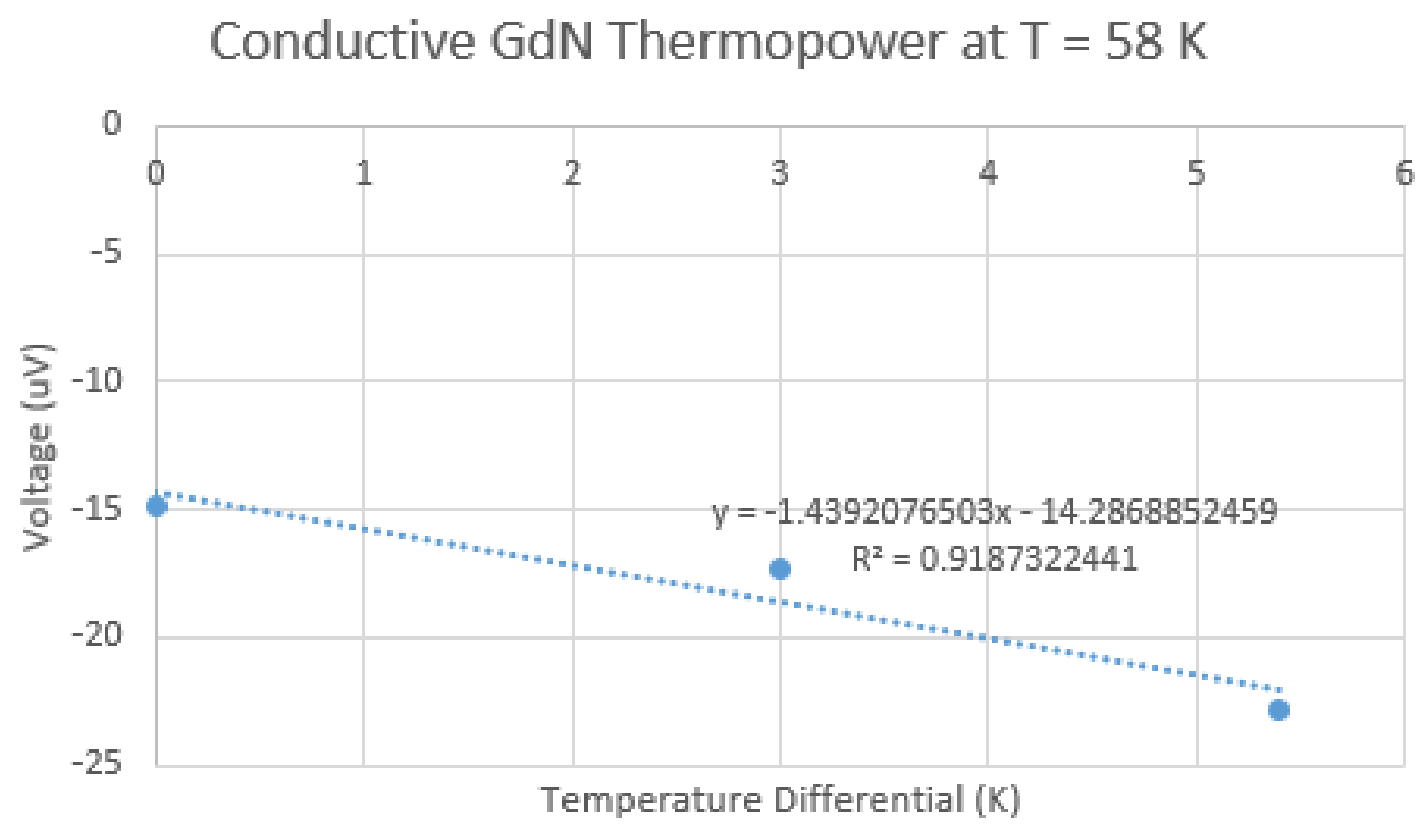

Figure 6.1: GdN Thermopower at $\mathrm{T}=58 \mathrm{~K}$

Conductive GdN Thermopower at $\mathrm{T}=83 \mathrm{~K}$

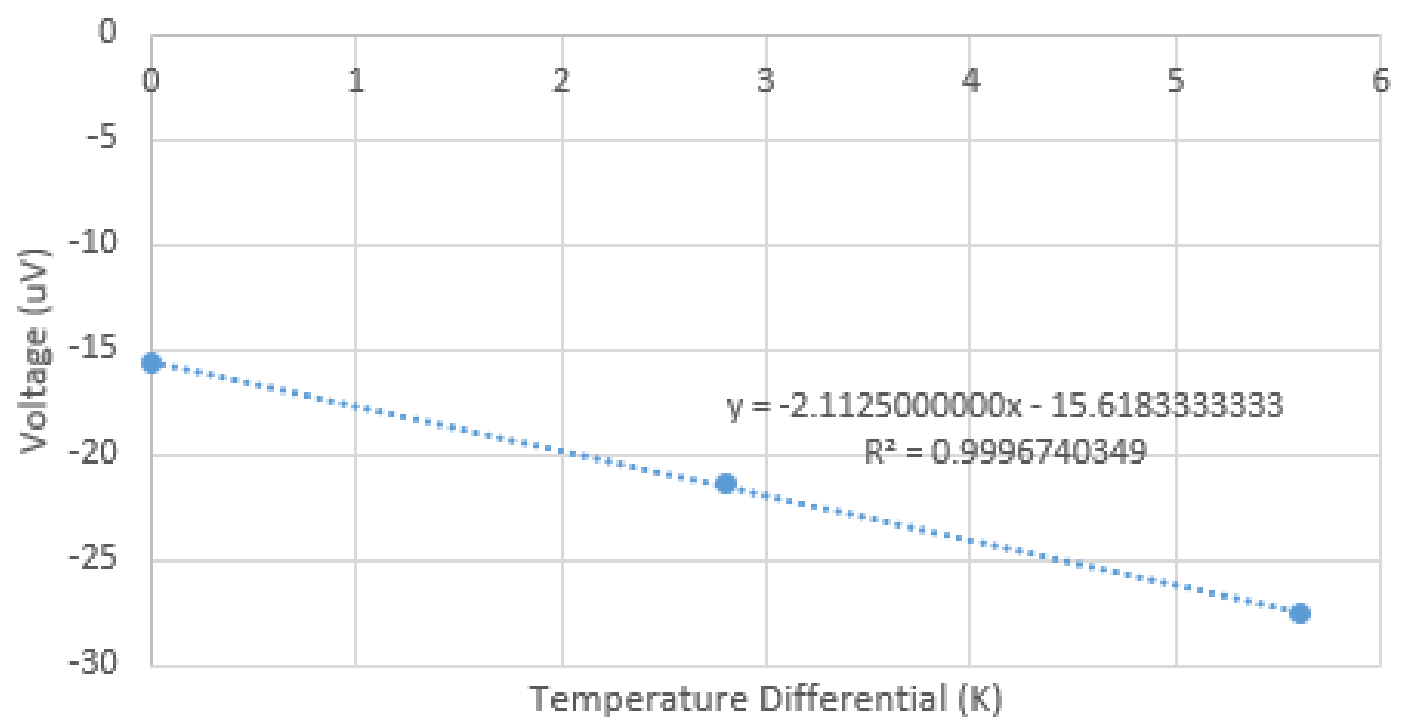

Figure 6.2: GdN Thermopower at $\mathrm{T}=83 \mathrm{~K}$ 
Conductive GdN Thermopower at T $=114 \mathrm{~K}$

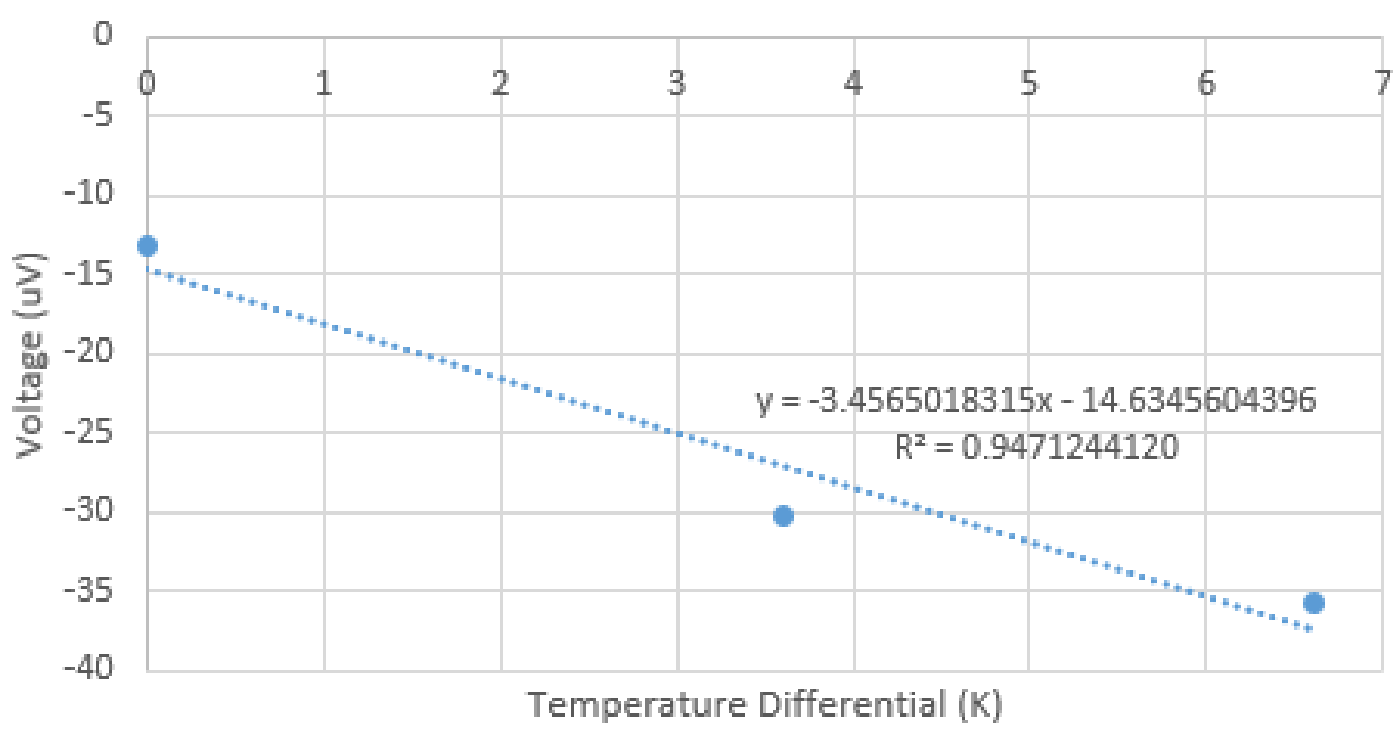

Figure 6.3: GdN Thermopower at $\mathrm{T}=114 \mathrm{~K}$

Conductive GdN Thermopower at T $=140 \mathrm{~K}$

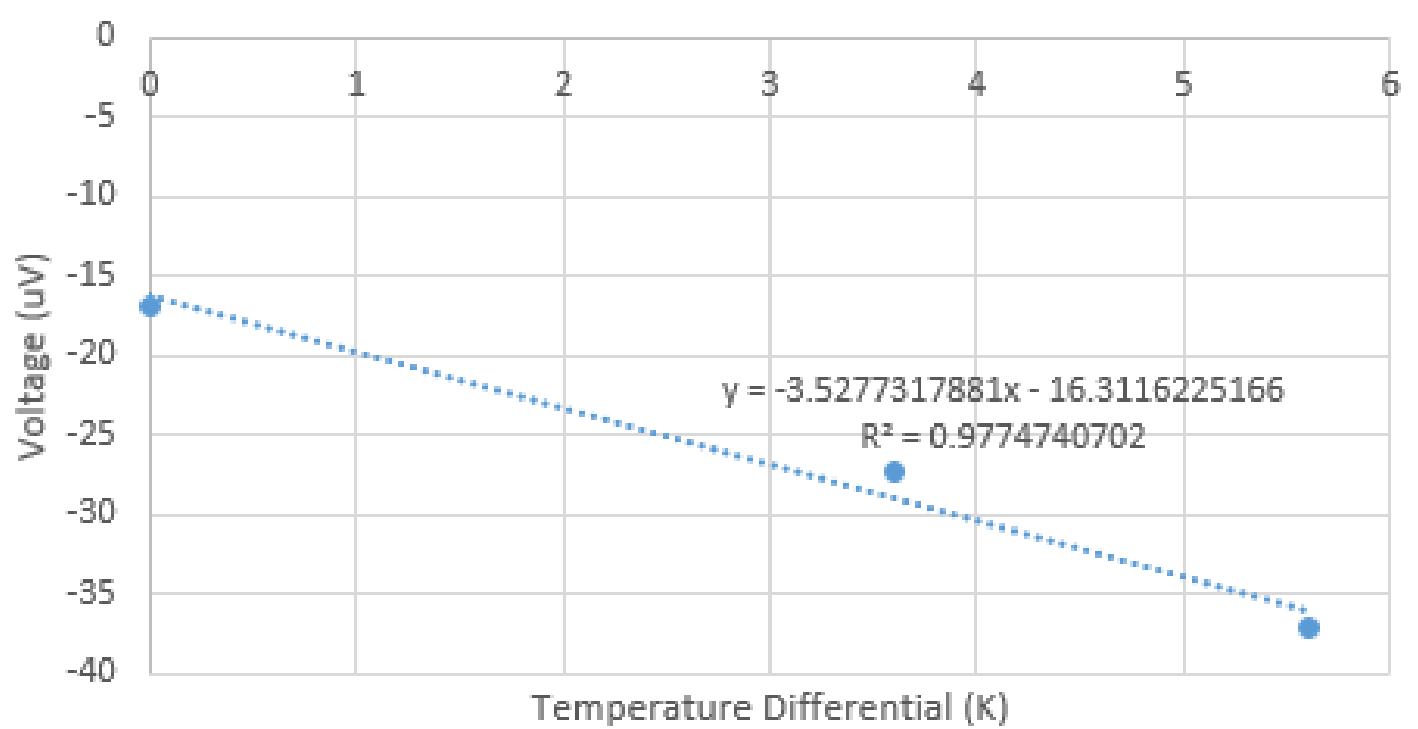

Figure 6.4: GdN Thermopower at $\mathrm{T}=140 \mathrm{~K}$ 
Conductive GdN Thermopower at T $=164 \mathrm{~K}$

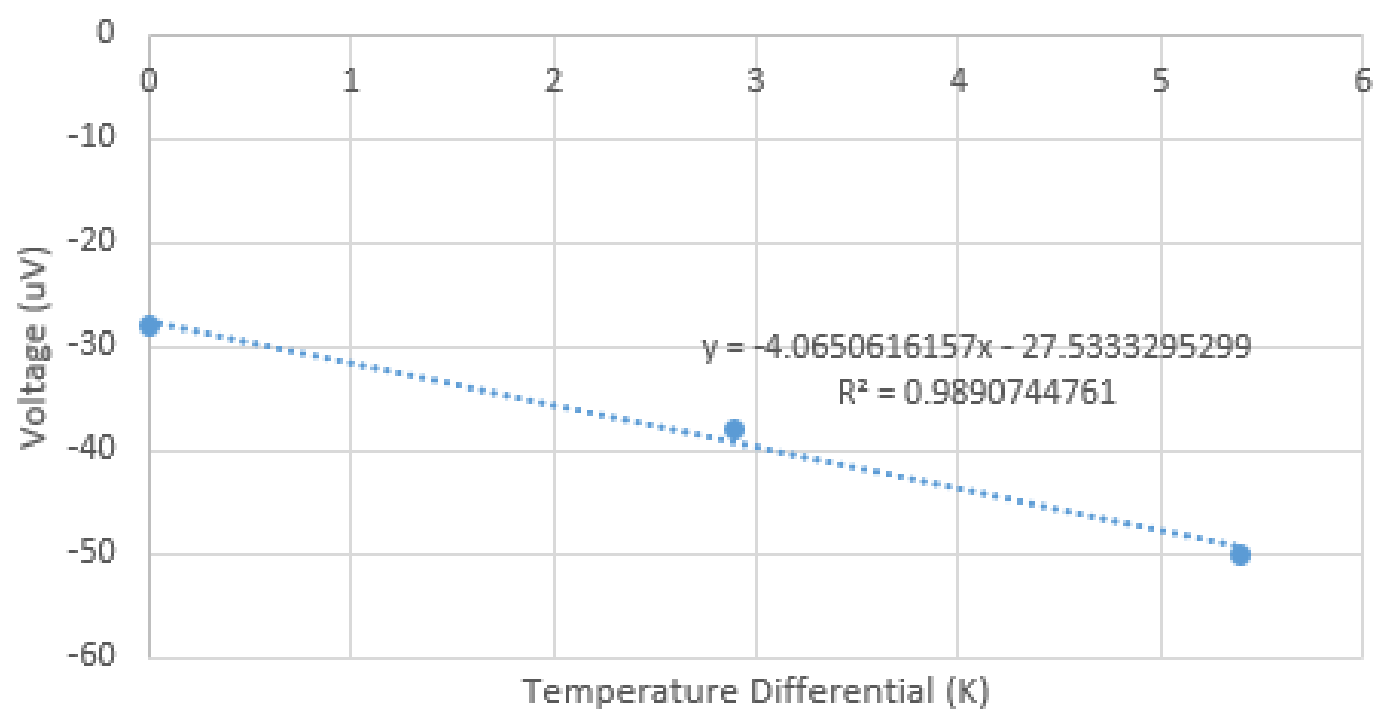

Figure 6.5: GdN Thermopower at $\mathrm{T}=164 \mathrm{~K}$

Conductive GdN Thermopower at $\mathrm{T}=180 \mathrm{~K}$

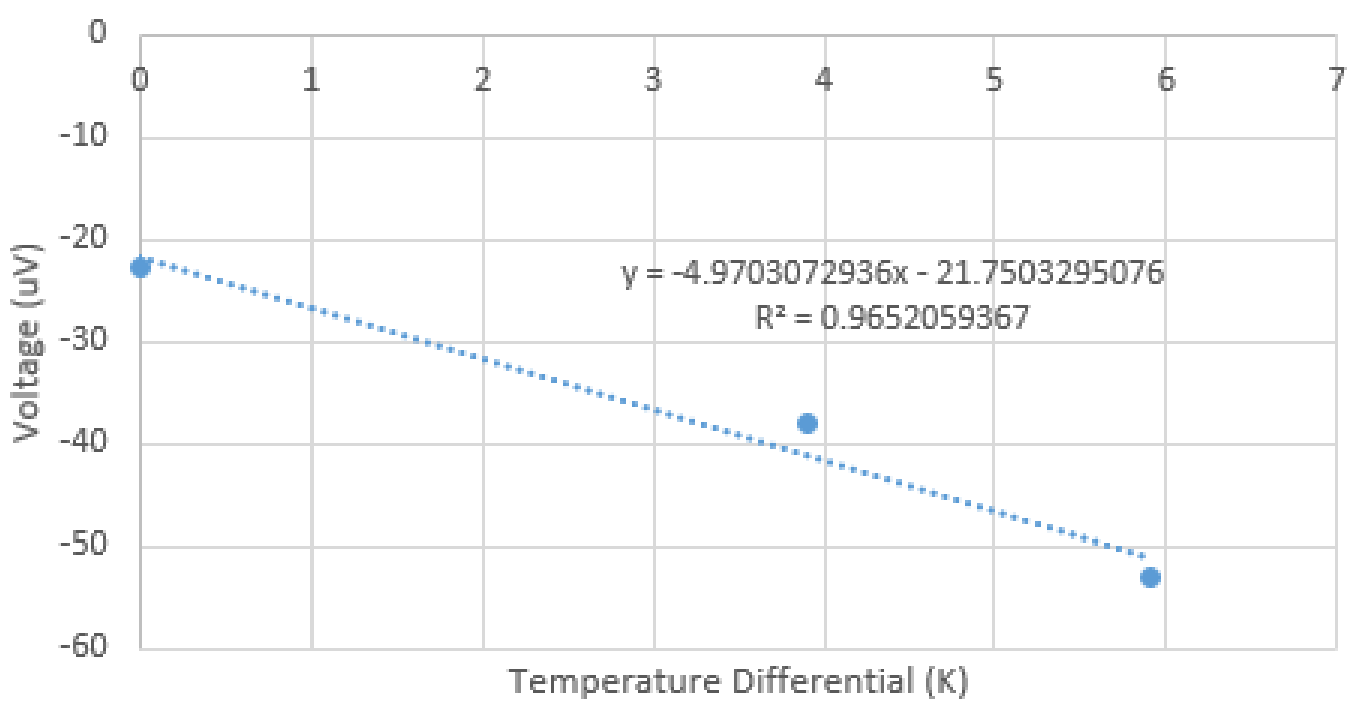

Figure 6.6: GdN Thermopower at $\mathrm{T}=180 \mathrm{~K}$ 


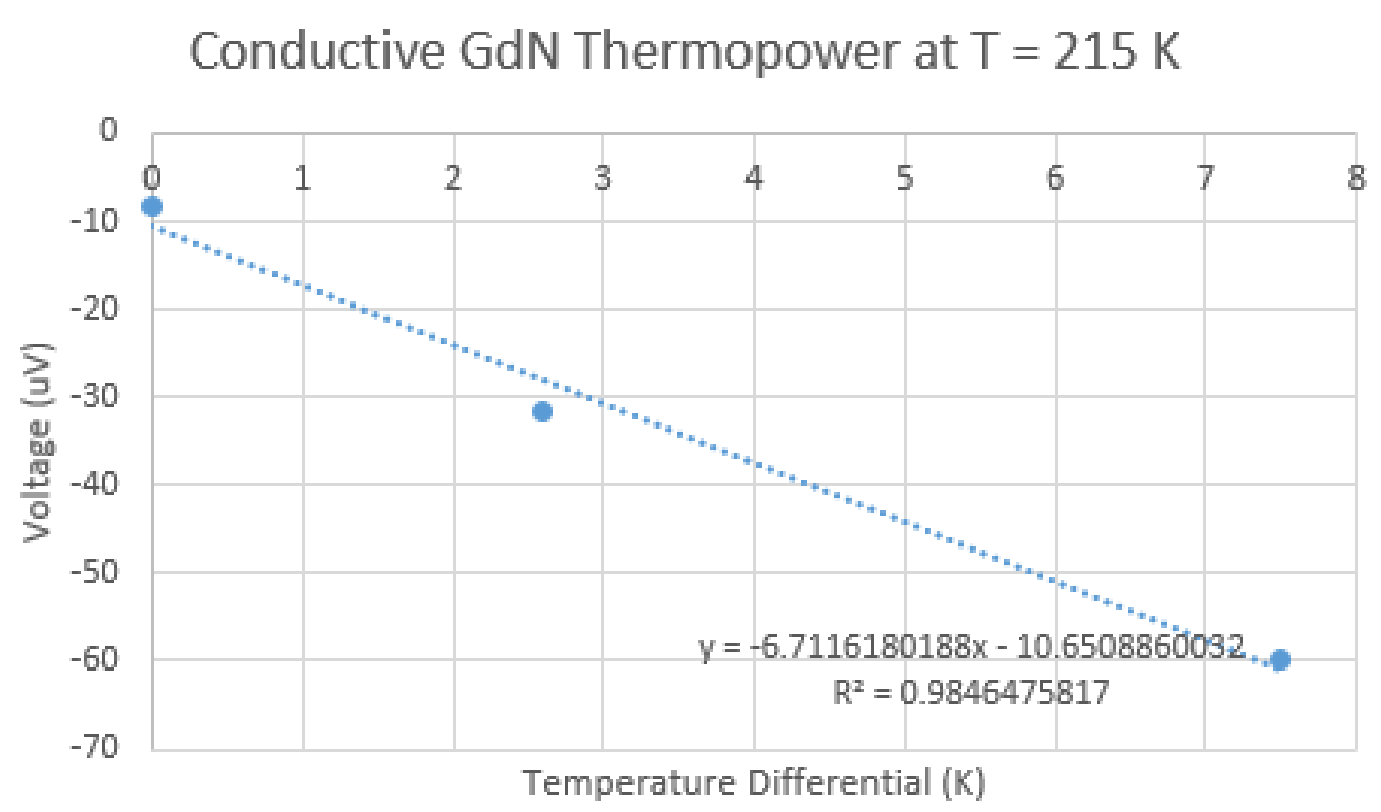

Figure 6.7: GdN Thermopower at $\mathrm{T}=215 \mathrm{~K}$

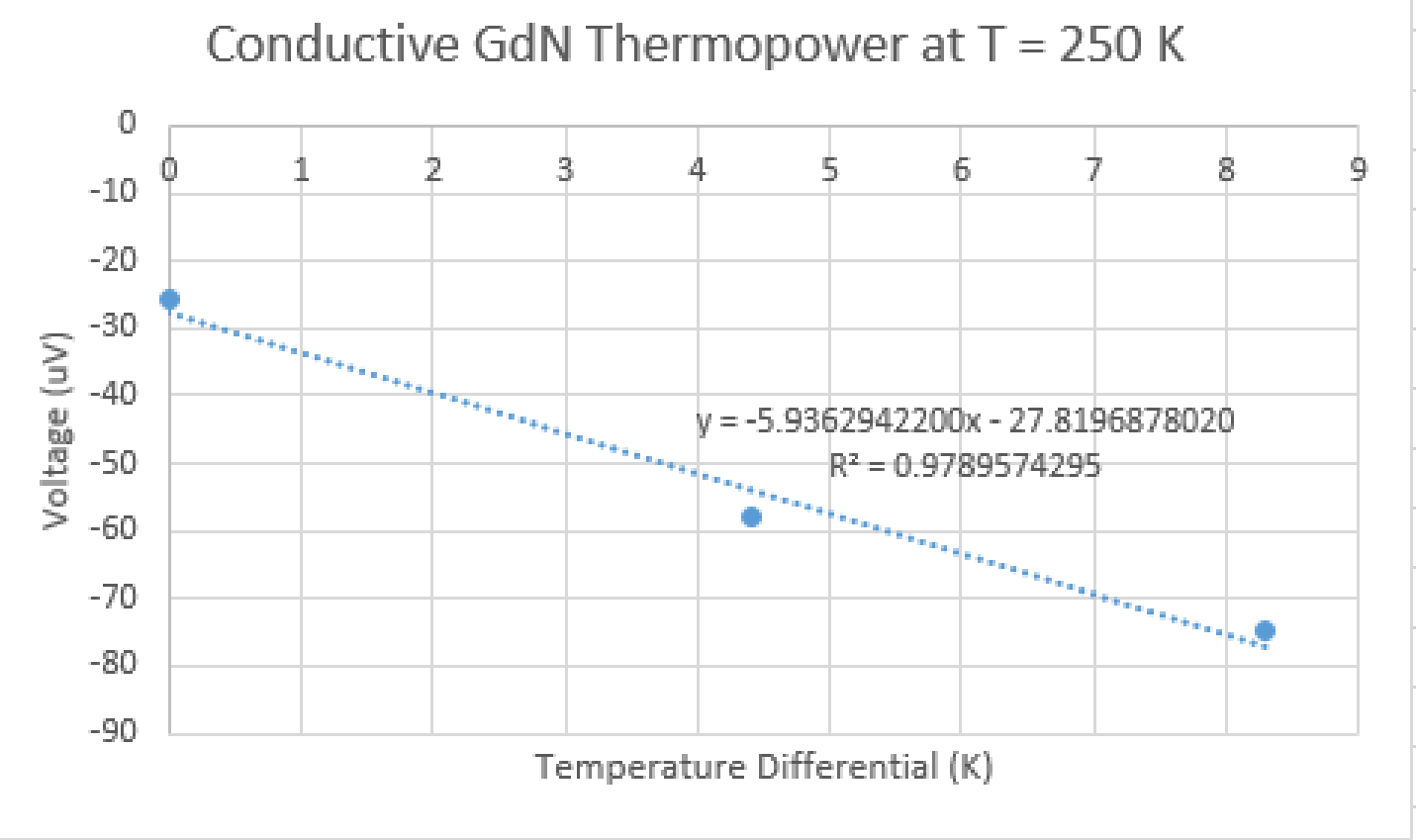

Figure 6.8: GdN Thermopower at $\mathrm{T}=250 \mathrm{~K}$ 


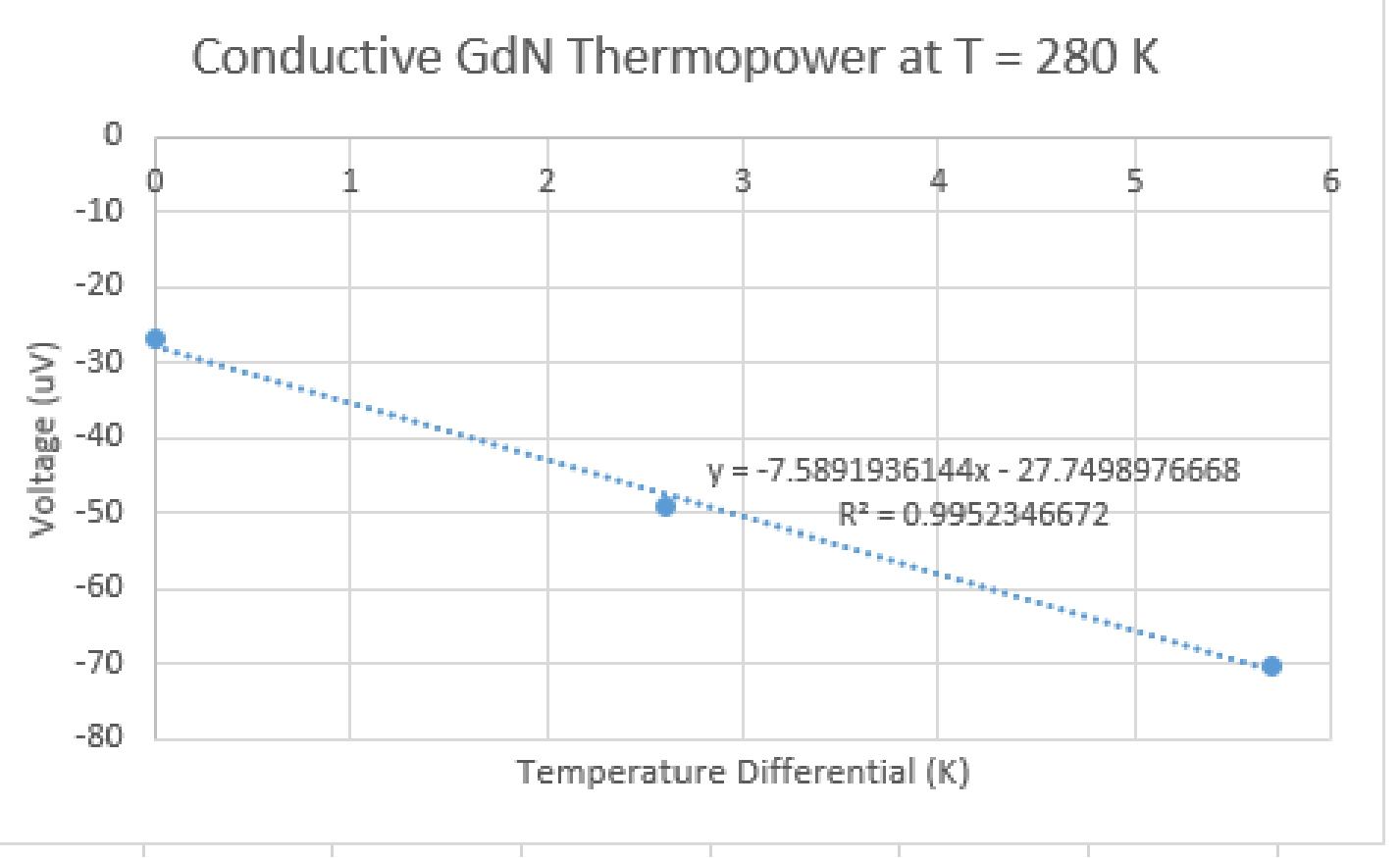

Figure 6.9: GdN Thermopower at $\mathrm{T}=280 \mathrm{~K}$

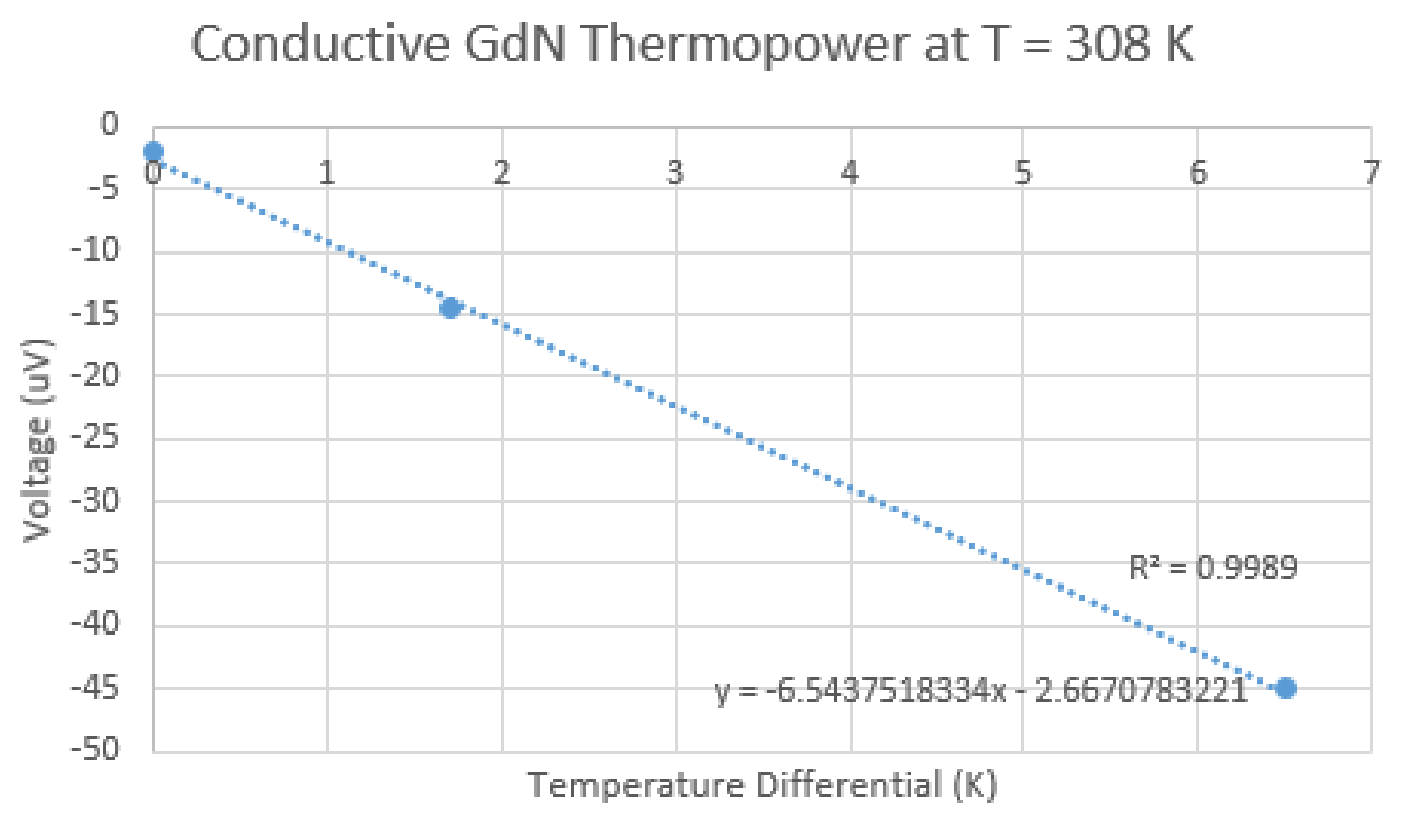

Figure 6.10: GdN Thermopower at $\mathrm{T}=308 \mathrm{~K}$ 


\section{Thermopower of GdN (w/out manganin correction)}

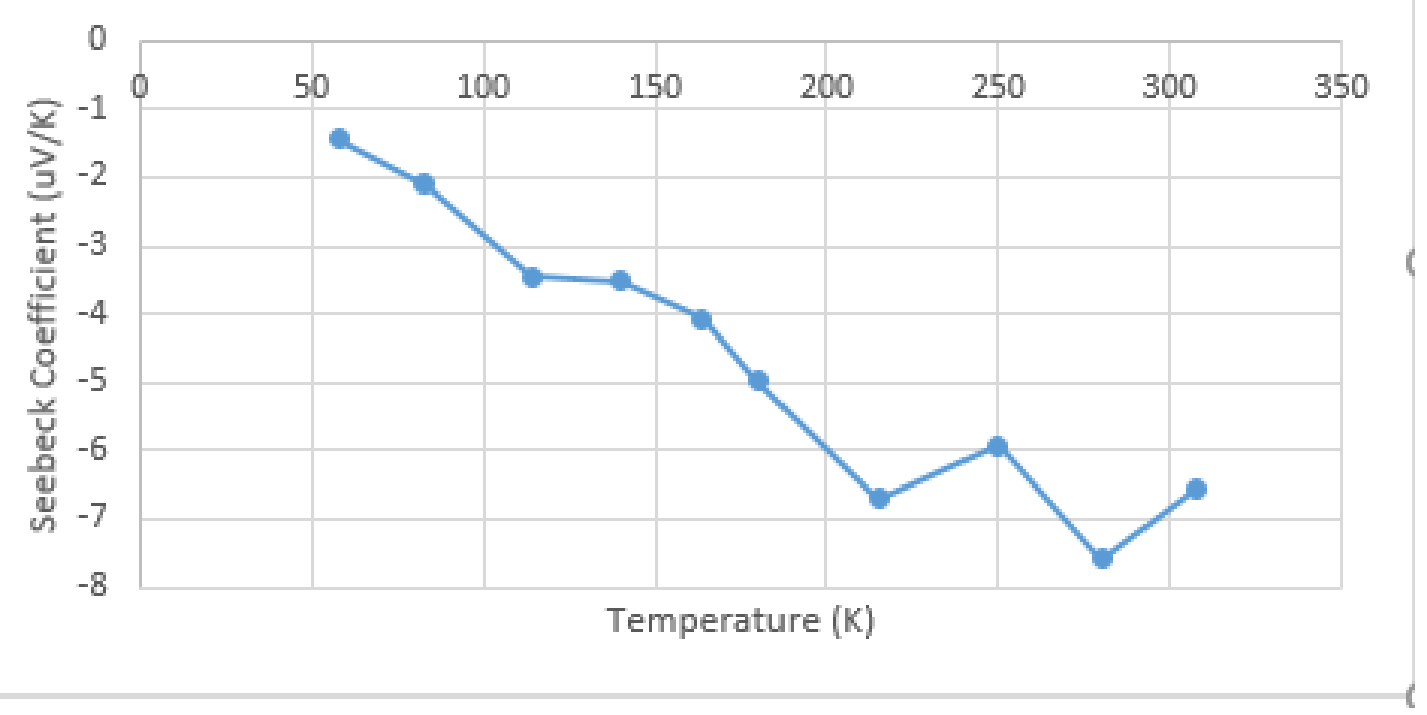

FiguRE 6.11: GdN Thermopower w/out manganin correction

Each of the above data plots contributes a single point to the final thermopower plot presented below. Figure 6.10 shows the thermopower of GdN without the corrections applied for the manganin leads, Figure 6.11 shows the thermopower of GdN with the manganin corrections, and Figure 6.12 demonstrates the experimental uncertainty values - magnified by 100x for ease of inspection. 
Thermopower of Gadolinium Nitride

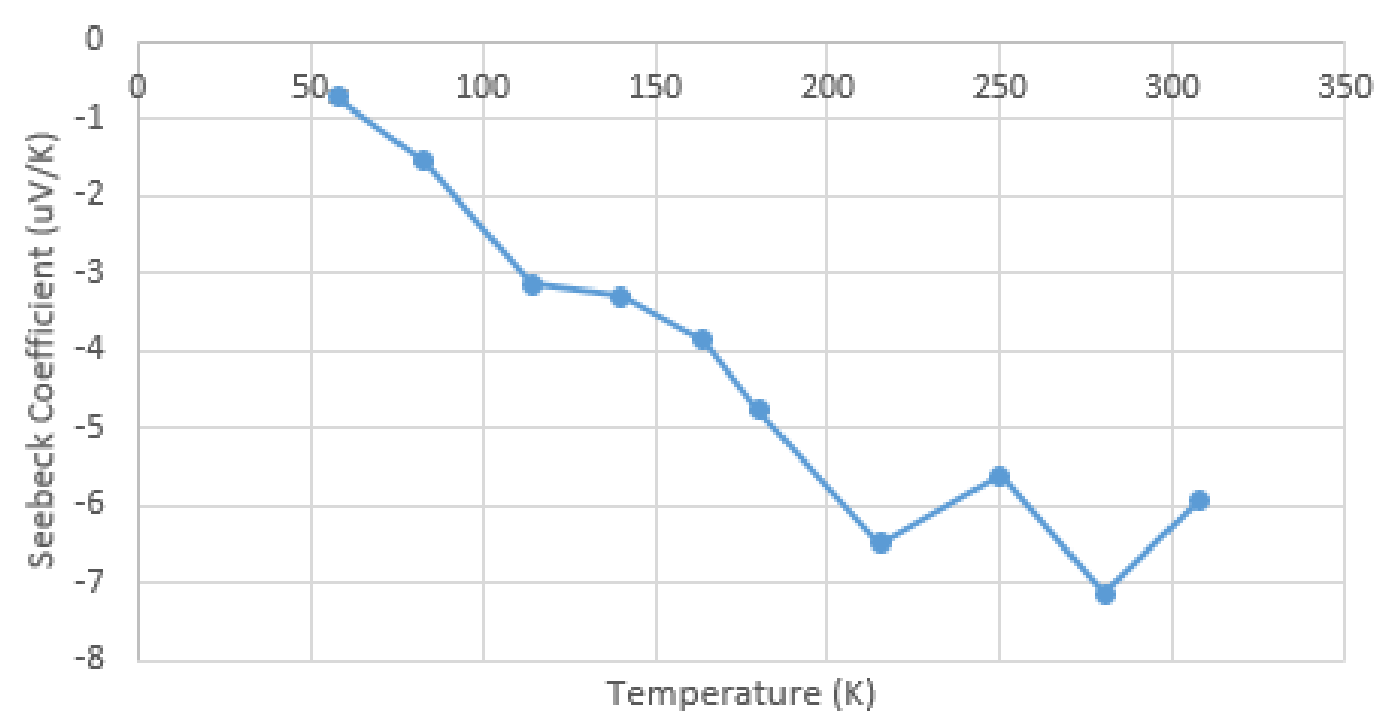

FiguRE 6.12: GdN Thermopower with manganin correction

\section{GdN Thermopower w/ 100*Uncertainty}

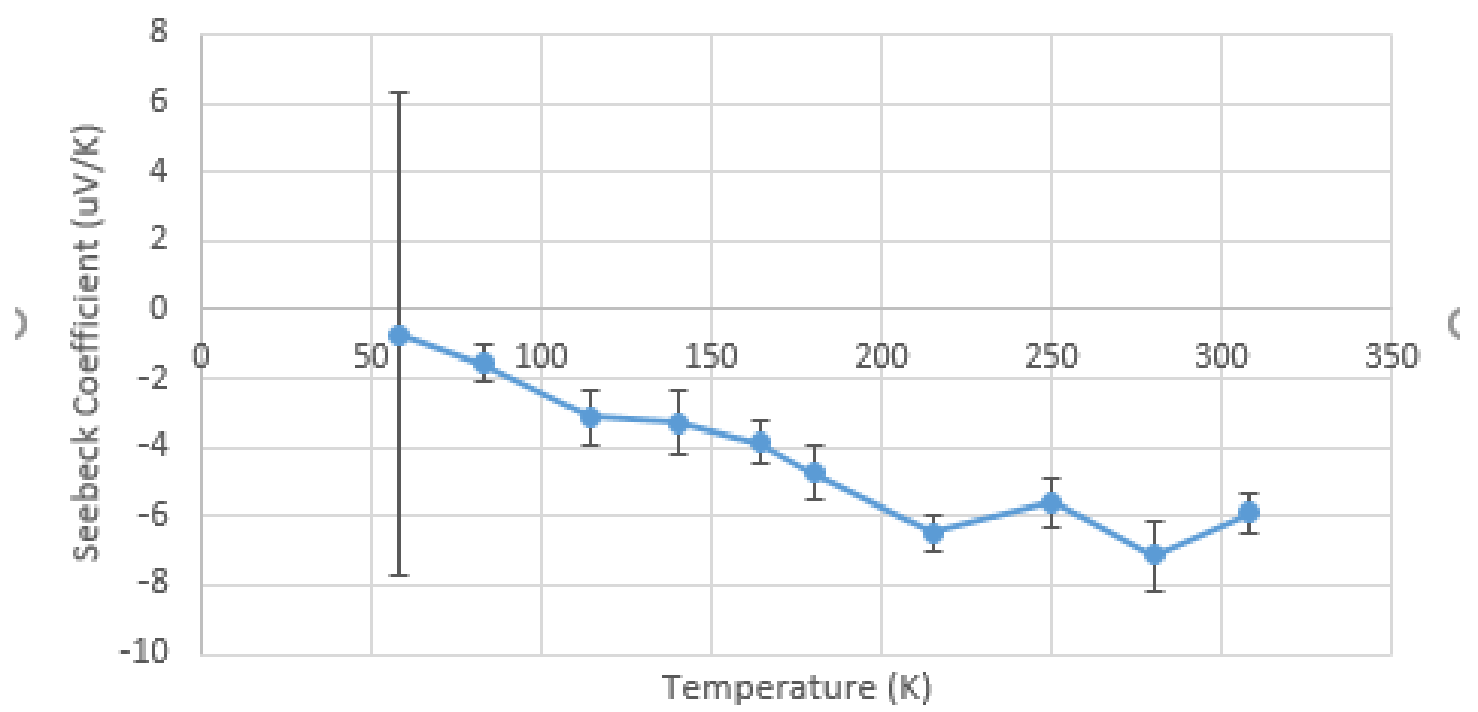

Figure 6.13: GdN Thermopower with $100^{*}$ uncertainty 
The thermopower of GdN is negative in sign. As far as it is shown here, the magnitude of the Seebeck coefficient for this sample tends to lie between $1 \mu \mathrm{V} \cdot \mathrm{K}^{-1}$ and $8 \mu \mathrm{V} \cdot \mathrm{K}^{-1}$. As temperatures fall towards $50 \mathrm{~K}$, the thermopower magnitude tends strongly towards zero. As temperatures rise from $50 \mathrm{~K}$ to $100 \mathrm{~K}$ the thermopower is enhanced, but the curve flattens as temperatures approach $110 \mathrm{~K}$. From $125 \mathrm{~K}$ to $225 \mathrm{~K}$ there is strong thermopower enhancement again, and as temperatures approach $300 \mathrm{~K}$ the thermopower begins to level off.

The thermopower trend appears to be roughly inversely proportional to temperature, with two notable departures - the thermopower measurements taken at $215 \mathrm{~K}$ and $280 \mathrm{~K}$ both show stronger results than what would otherwise be suggested by the trend in the data. These departures from the expected trend are not explained or mitigated by changes in the manganin thermopower as indicated by the lack of differences between Figure 6.10 and Figure 6.11.

Sample resistance measurements were also taken during the thermopower measurement process. Although they are inferior to those obtainable via the 4-pole method with the REN Group's closed-cycle cryostat, getting the extra measurements was comparatively simple and brings extra insight to the interpretation of final results. The resistivity ( $\rho=A R / L$ ) is calculated using the sample resistance $R$, the conductive cross-section $A=w t$ where $w$ is the sample width and $t$ the thickness, and the sample length $L$. 


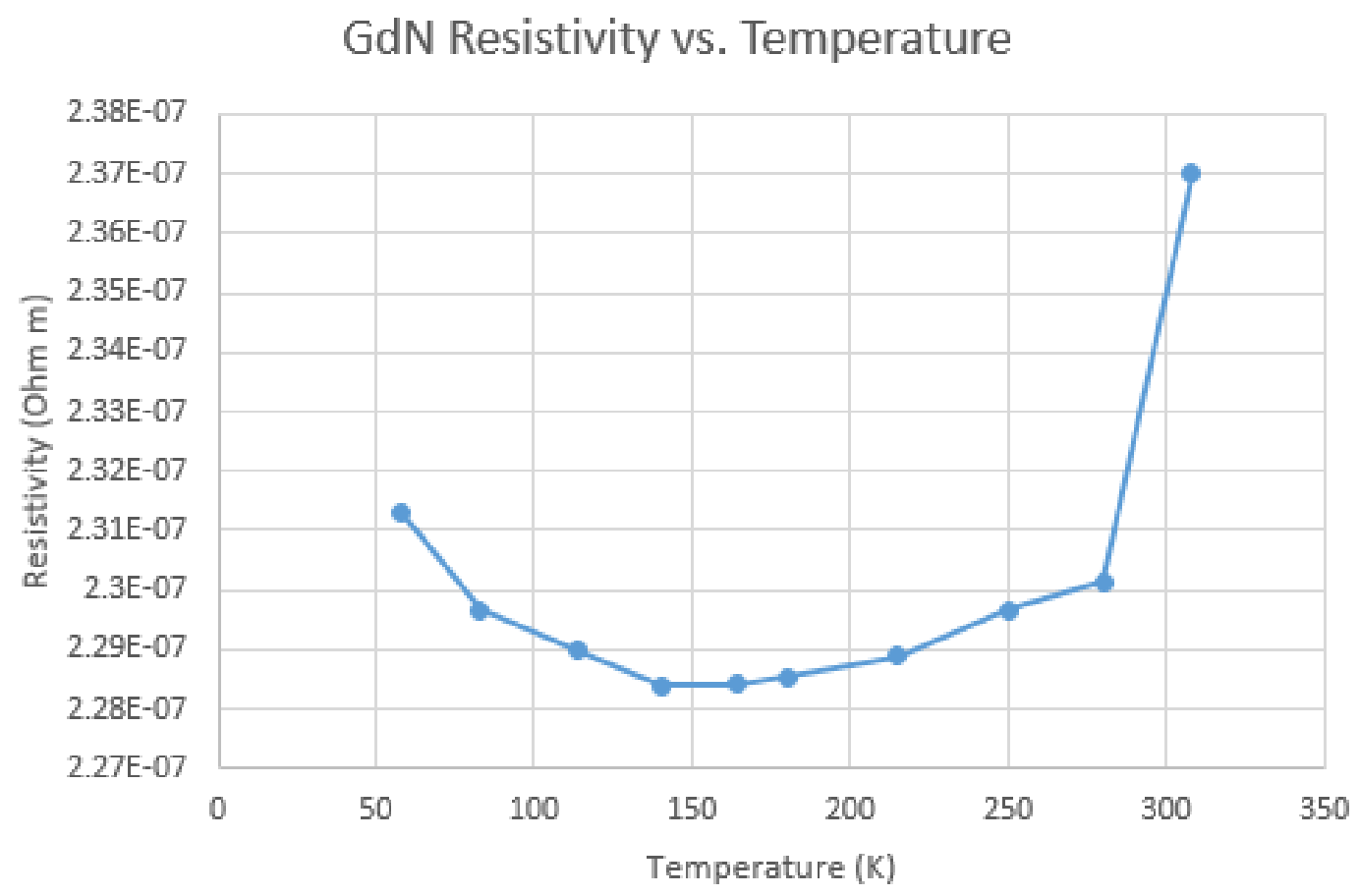

FiguRE 6.14: GdN Resistivity vs. Temperature

The resistivity data aboves shows this sample of GdN's resistivity varying from a minimum of $2.285 \times 10^{-7} \Omega \cdot \mathrm{m}$ at $T=150 \mathrm{~K}$ to a maximum of $2.37 \times 10^{-7} \Omega \cdot \mathrm{m}$ at $T=$ $308 \mathrm{~K}$.

It is known that the measured thermopower response is primarily composed of phonon drag thermopower (scaling with $T$ ) and charge diffusion thermopower (scaling with $T^{3}$ ). Therefore we expect a thermopower vs. temperature curve to display minimal response near $0 \mathrm{~K}$, a maximum and flattening response at higher temperatures, and a region between the two showing a trade off between $T$ and $T^{3}$ dependence. The charge carrier diffusion component will always give a thermopower contribution of the same sign as the charge carried, but the phonon drag component may give a positive or negative contribution depending on the temperature, and the trade off between energy state availability above and below the Fermi level. These data are roughly linear and clearly negative which suggests that as temperatures fall a negative phonon drag component begins to contribute where the electron diffusion component proportional to $T^{3}$ otherwise tends toward zero.

Overall, these data agree well with two of the predictions made by the researchers - that the sign of the thermopower would match that of the charge carriers (negative), and that the magnitude would typically be of an order of magnitude above the thermopower 
values seen in pure metals. However, time constraints as well as sample production difficulties prevented the researchers from obtaining samples with a usable range of nitrogen vacancy concentrations, which meant that the prediction of a maximised thermopower response under a light- to -medium level of doping could not be verified at this stage.

\subsection{Error Analysis}

The experimental uncertainties associated with the sample voltage measurements themselves are tightly constrained (typically less than $\pm 0.1 \%$ ). The highly precise Tektronix multimeter and the reliable connections formed by the indium tips provided consistent and reliable data. Therefore experimental noise is not sufficient to explain the clear trend departure made by the points at $T=215 \mathrm{~K}$ and $T=280 \mathrm{~K}$. These two points were not measured in the same cooling/heating run as for the rest of the data. They were added later on a subsequent cooling run after the sample and cryostat had rested for several days. When the sample space pressure was checked again, it was discovered that it had leaked and admitted air during that time. If the sample had oxidised in response to air during this period, the increased number of impurities and crystal dislocations per unit volume would add extra charge scattering mechanisms. It is known both from theory and experiment that such additional scattering mechanisms have a greater effect on the thermopower than on the resistivity (which showed no obvious accompanying increase), thus, it is the opinion of the researchers that the trend departures seen at $T=215 \mathrm{~K}$ and at $T=280 \mathrm{~K}$ are the result of oxidation-induced crystal domain dislocations causing additional electron scattering at their boundaries.

A second source of error comes from our temperature measurements. The Lakeshore DT-670 Si-diode thermometers are precise and of high build quality, with a minimum response voltage of $10 \mu \mathrm{V}$ but the multimeters available to the researchers for temperature tracking could only read a minimum response of $1 \mathrm{mV}$. Therefore a temperature differential as large as $0.6 \mathrm{~K}$ could be in place before such multimeters could make the researchers aware. Attempts to wash this source of error out of the data were made by using larger values for $\Delta T$ but too large a $\Delta T$ begins to increase uncertainty again as the assumption of thermopower response linearity over $\Delta T$ gets weaker.

A third source of error comes from the power control to the heaters. Due to equipment constraints, the power sources for the $26.5 \Omega$ differential heater and the $80 \Omega$ stage heater were of analogue design and controlled with dials. They had a much greater range and much less fine control than what would have been ideal. This meant that, rather than adjusting and controlling the heater power to achieve a desired $\Delta T$, a differential 
heater power was chosen and allowed to stabilise after which the $\Delta T$ across the sample was calculated. The lack of fine current control meant that it was difficult to obtain more than three thermopower data points at each temperature measurement.

Finally, one might have noticed the consistently non-zero y-intercept values on the $V_{s}$ vs. $\Delta T$ measurements. In theory, these intercepts should all be zero. There are a number of thermal gradients and dissimilar metal junctions within the experimental apparatus, each of which provides a thermoelectric contribution. The largest contributor will be the constantan wires connecting the sample stage to the multimeters. Constantan has its own non-zero thermopower response and on average this response will grow when the temperature difference between the sample environment and the lab environment grows larger. However, constantan's nonlinear thermopower means that its overall contribution isn't maximised when the temperature differential between sample and lab space is maximised. In fact, the largest non-zero intercept was $-28.15 \mu \mathrm{V}$ seen at $T=164 \mathrm{~K}$. A rough calculation using these numbers gives a constantan thermopower response on the order of $200 \mathrm{nV} \cdot \mathrm{K}^{-1}$ which agrees well with the referenced literature concerning manganin's thermopower. This makes sense considering that both constantan and manganin are copper-nickel alloys engineered for their relatively low thermal conductivity.

\subsection{Summary}

- The thermopower of GdN was negative in magnitude over the whole range investigated, as was expected of a high nitrogen-vacancy rare-earth nitride.

- The highest thermopower response was seen at high temperatures, and the response was minimal as temperature tended towards zero - both observations were predicted by theory.

- Two points showed a thermopower response higher than the plot trend would otherwise indicate. These points were obtained 6 days after the rest of the data set. The increased thermopower response is interpreted as oxidation-induced crystal deformations causing additional electron scattering at their margins.

- There was no accompanying change in resistivity at these points. It is known that impurities, dislocations, and other such scattering mechanisms have a greater effect on thermopower measurement than they do on resistivity measurements. 


\subsection{Future Work}

There is much scope for improvement of our understanding of gadoliunium nitride, of rare-earth nitrides, and of thin-film semiconductors in general. Thermopower is just one of the many tools that aid the researcher in their investigations.

\subsubsection{Instrumental Improvements}

- Fix cryostat seals to preserve samples for longer.

- Consider replacing cryostat in order to make liquid helium temperatures available again.

- Get a low-power, high-precision current controller with dual output to enable simultaneous control of sample and stage temperatures.

- Get two more Tektronix 6.5 digit precision multimeters to replace the handheld multimeters currently in use. Combined with the high-precision current controller they will offer much greater accuracy and control of thermopower measurements.

- Tap two new wire ports into the top of the removable sample stage to allow uninterrupted manganin connections straight from sample to multimeter. This removes the additional thermoelectric effects seen at dissimilar metal junctions.

\subsubsection{Further Investigations}

There are three main directions further research on the thermopower of GdN could take:

1. Devote more time and research to the sample production process, in order to more tightly control nitrogen vacancy concentration.

A deeper understanding of exactly how the growth process parameters affect final nitrogen vacancy concentrations could help us end up with a large number of samples showing a wide range of nitrogen vacancies and associated resistivity measurements. It is believed that thermopower and electrical resistivity will correlate strongly in rare-earth nitrides and there is much scope for investigation of this relationship in the future. 
2. It is known that the phonon-drag component of thermopower is affected by the presence of an external magnetic field.

Rebuilding the sample stage in order to fit it inside the magnet would require a significant amount of work and technical expertise. However, doing so would allow future researchers to add two new experimental parameters to their investigations magnetic field strength, and direction. Repeated thermopower measurements over the whole available temperature range while varying either magnetic field strength or orientation should show a strong effect on the thermopower region most strictly governed by phononic effects.

3. Thermopower measurements as an indicator of impurities or oxidation.

During this research, sample oxidation caused a notable increase in thermopower magnitude while leaving the resistivity measurements unchanged. While such sample oxidation is typically nothing more than an annoying nuisance for the researcher, it is possible that repeated thermopower measurements could be used to track sample oxidation over time. These data could then be used to evaluate the efficacy of different passivation layers on thin-film samples. Such information could help other scientists working in the field of molecular-beam-epitaxy to produce longer-lasting and more reliable samples. 
Appendix A

Data and Calculations 


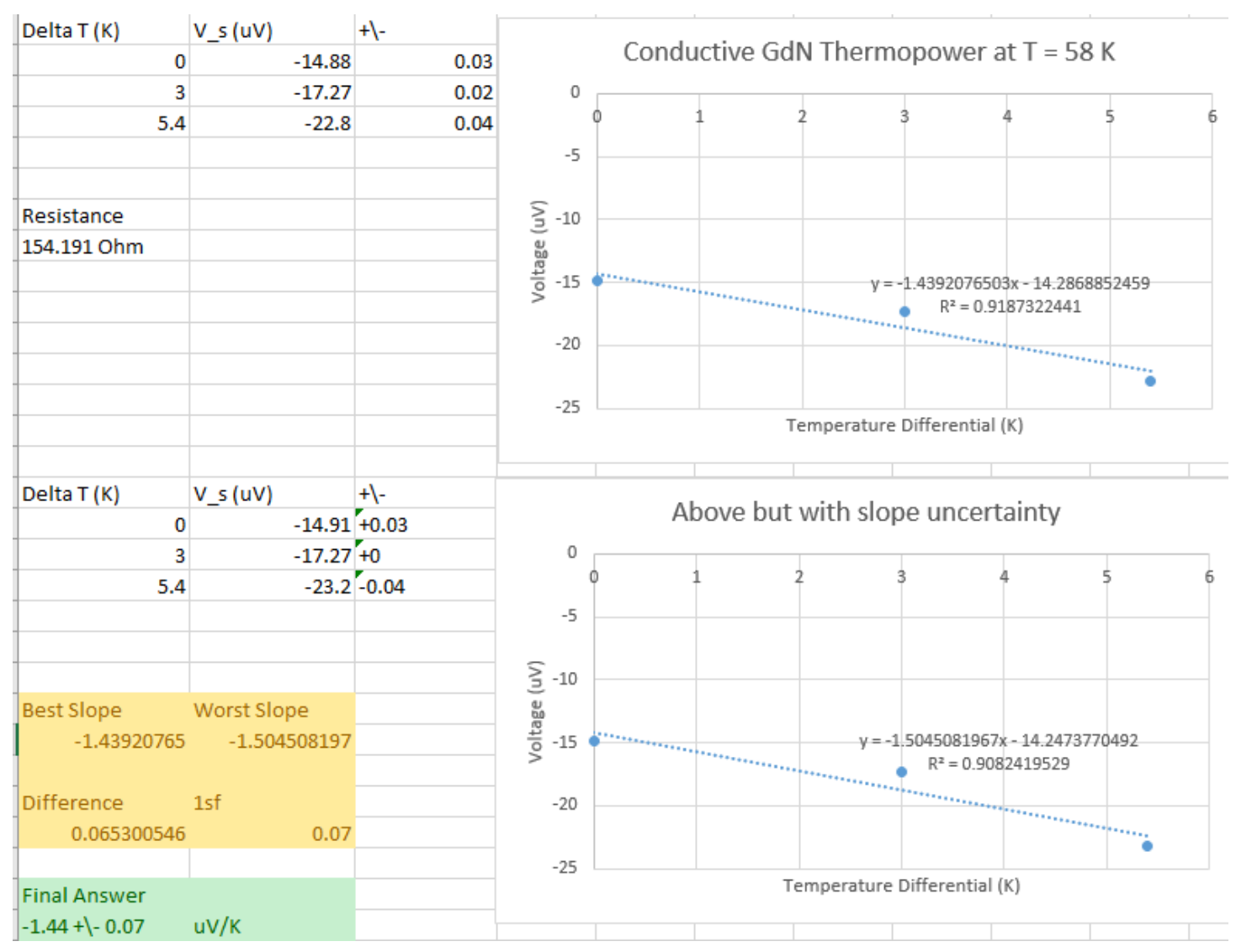

Figure A.1: Thermopower calculations at $\mathrm{T}=58 \mathrm{~K}$ 


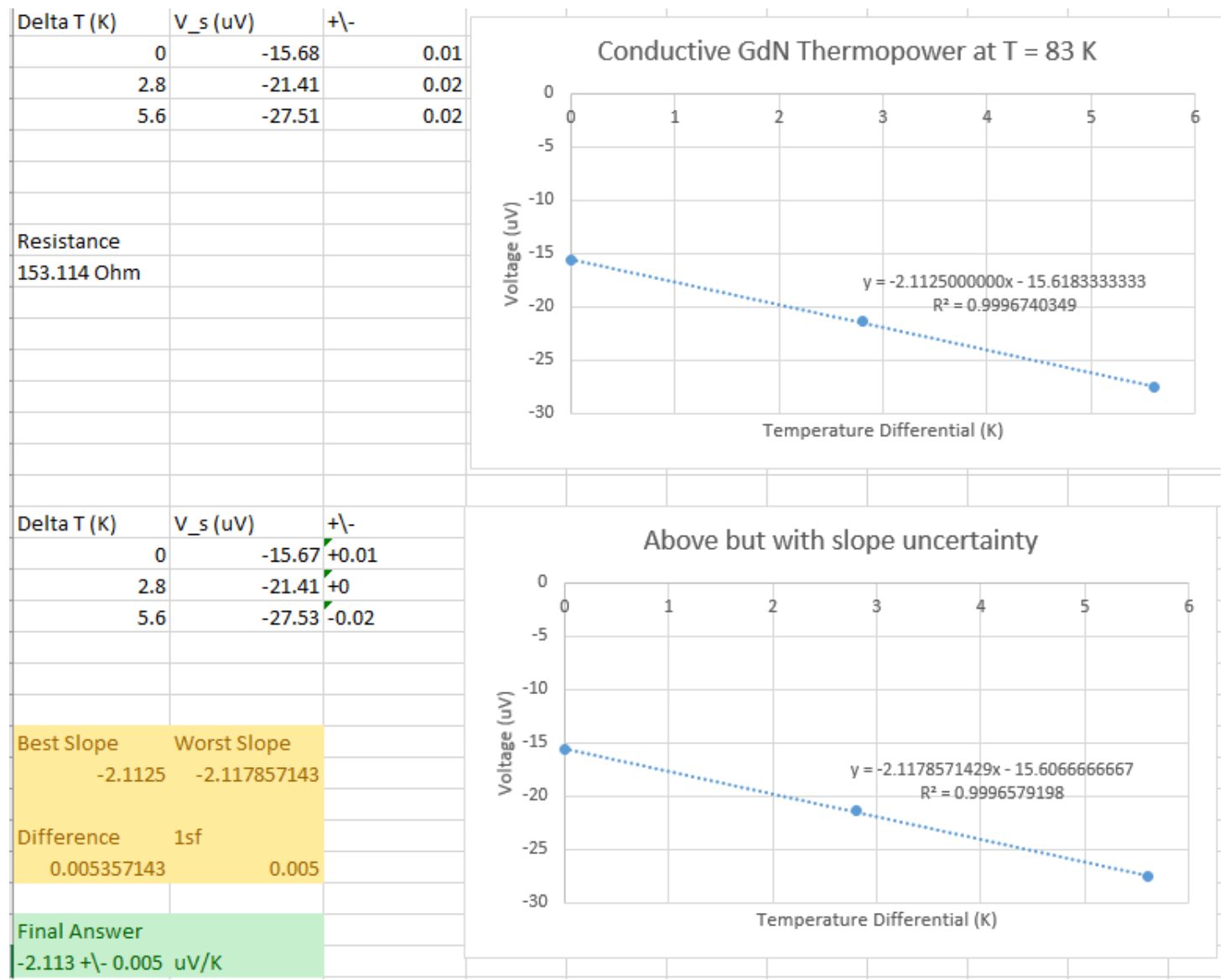

Figure A.2: Thermopower calculations at $\mathrm{T}=83 \mathrm{~K}$ 


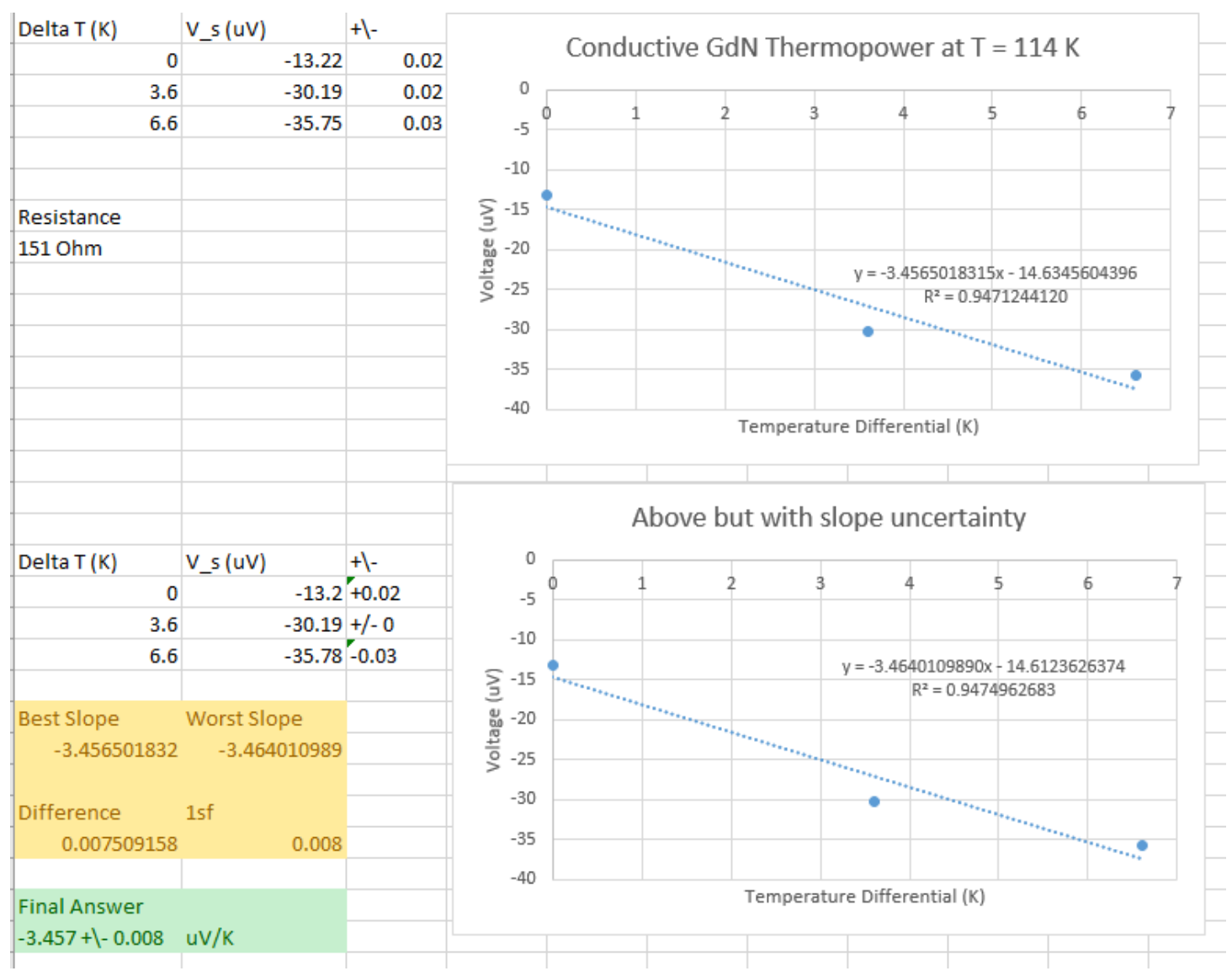

Figure A.3: Thermopower calculations at $\mathrm{T}=114 \mathrm{~K}$

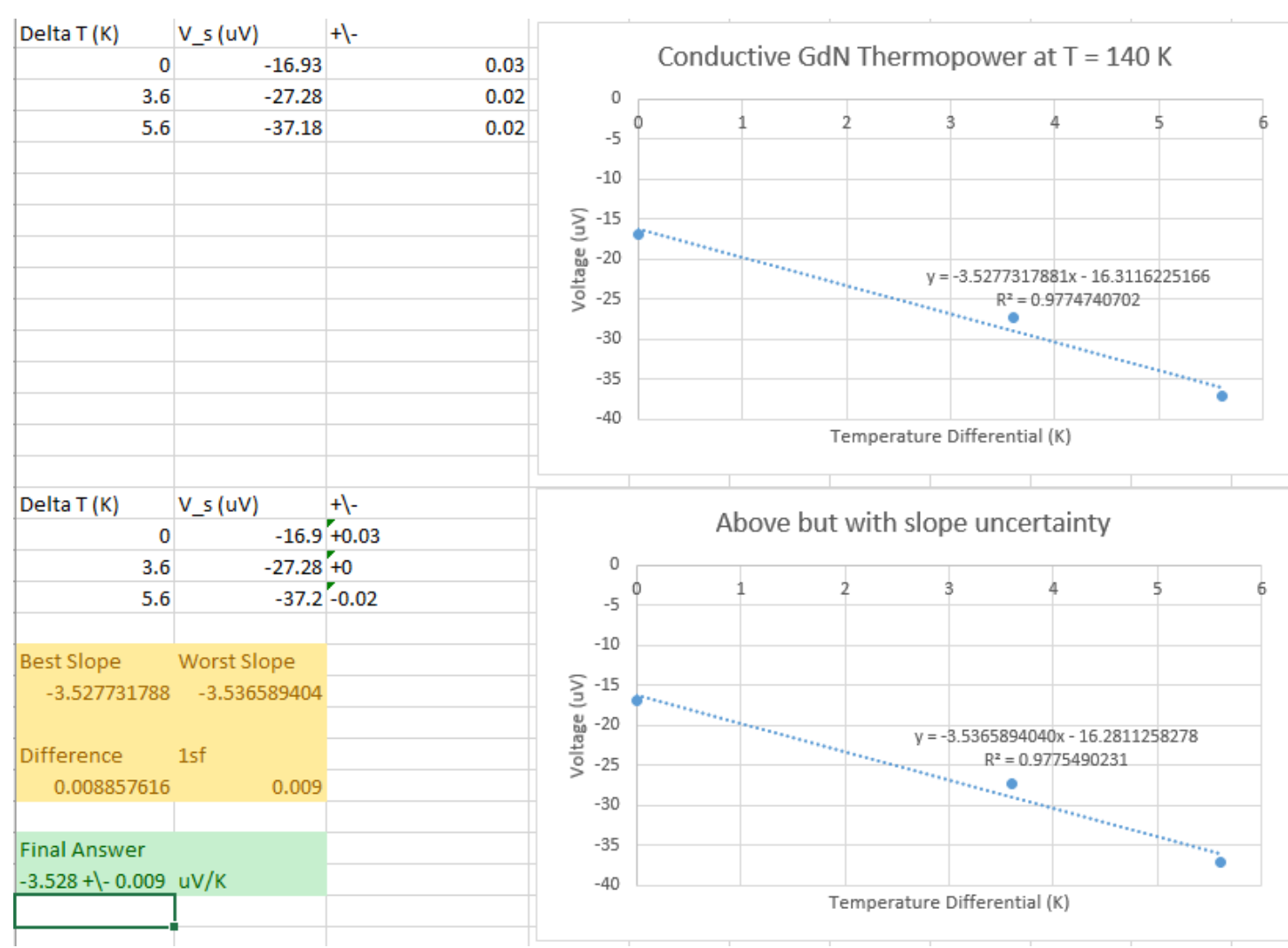

Figure A.4: Thermopower calculations at $\mathrm{T}=140 \mathrm{~K}$ 

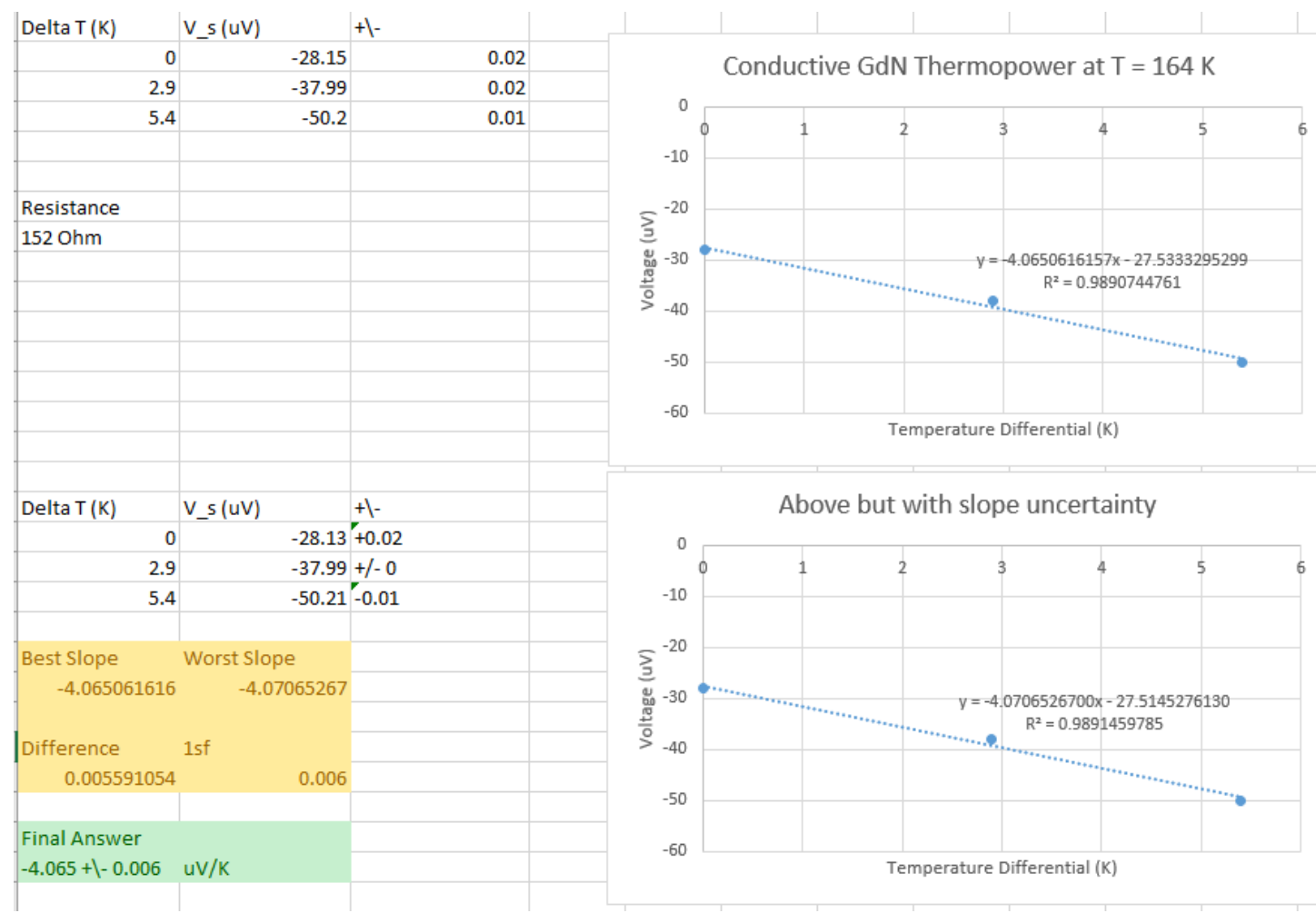

Figure A.5: Thermopower calculations at $\mathrm{T}=164 \mathrm{~K}$

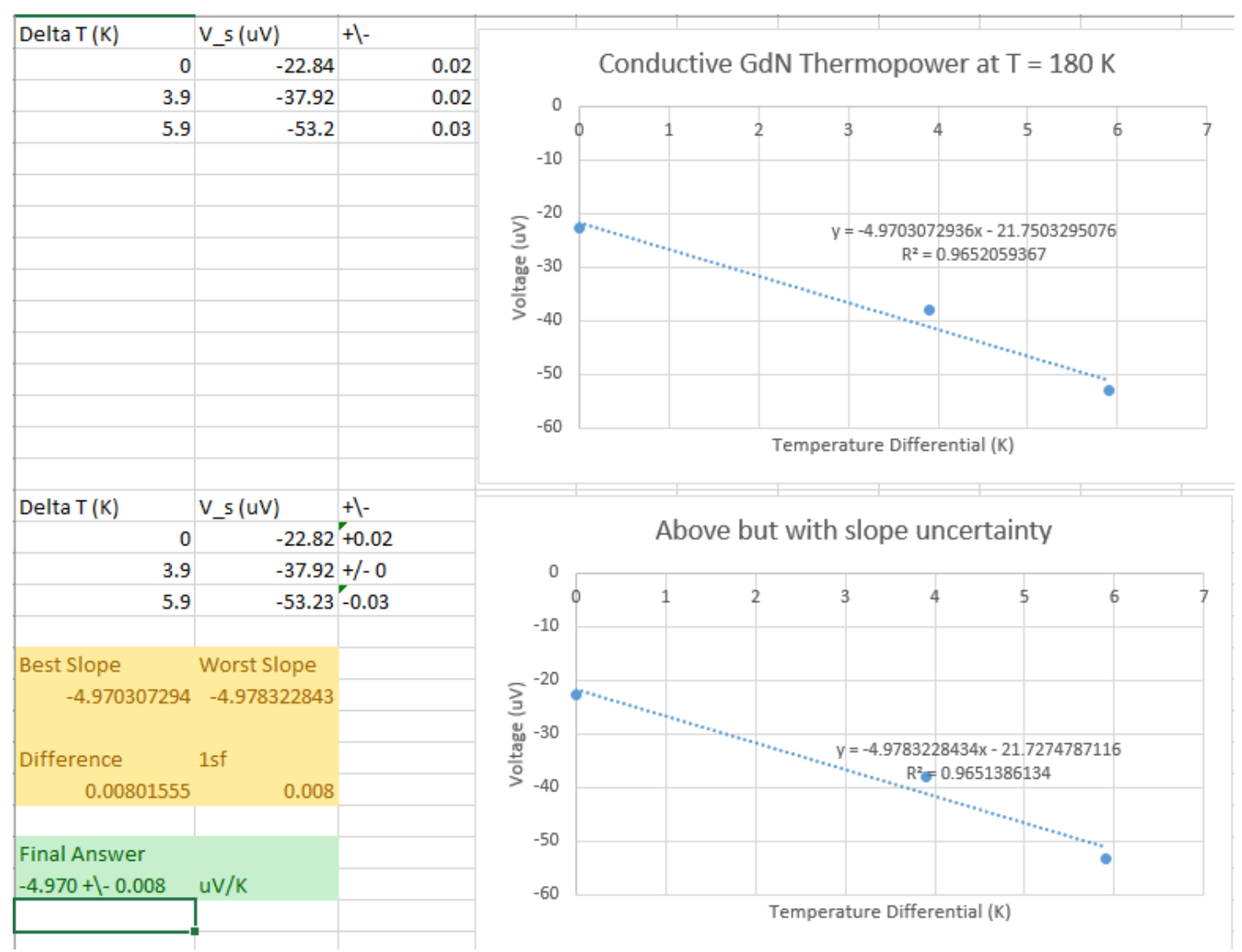

Figure A.6: Thermopower calculations at $\mathrm{T}=180 \mathrm{~K}$ 


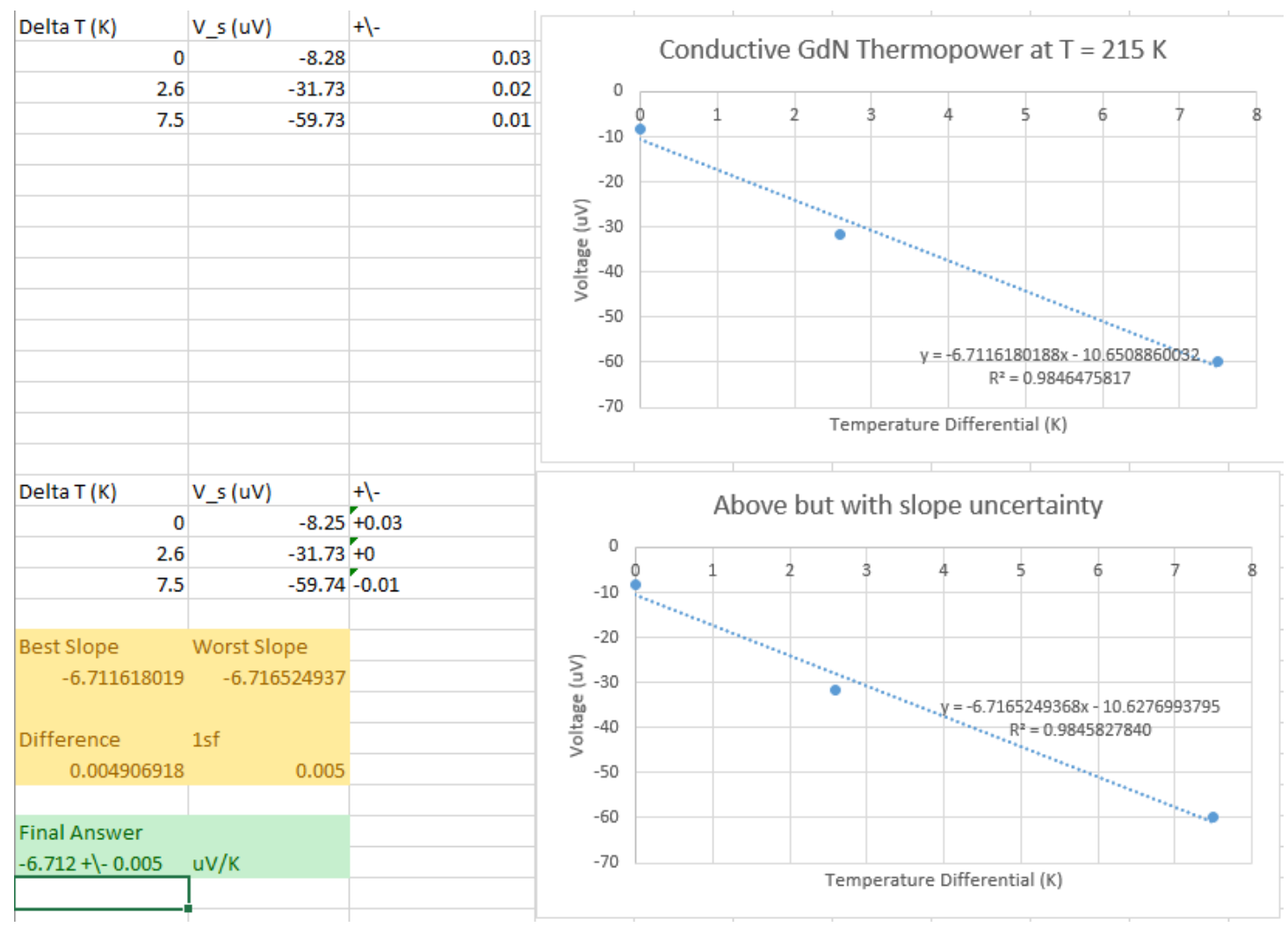

Figure A.7: Thermopower calculations at $\mathrm{T}=215 \mathrm{~K}$

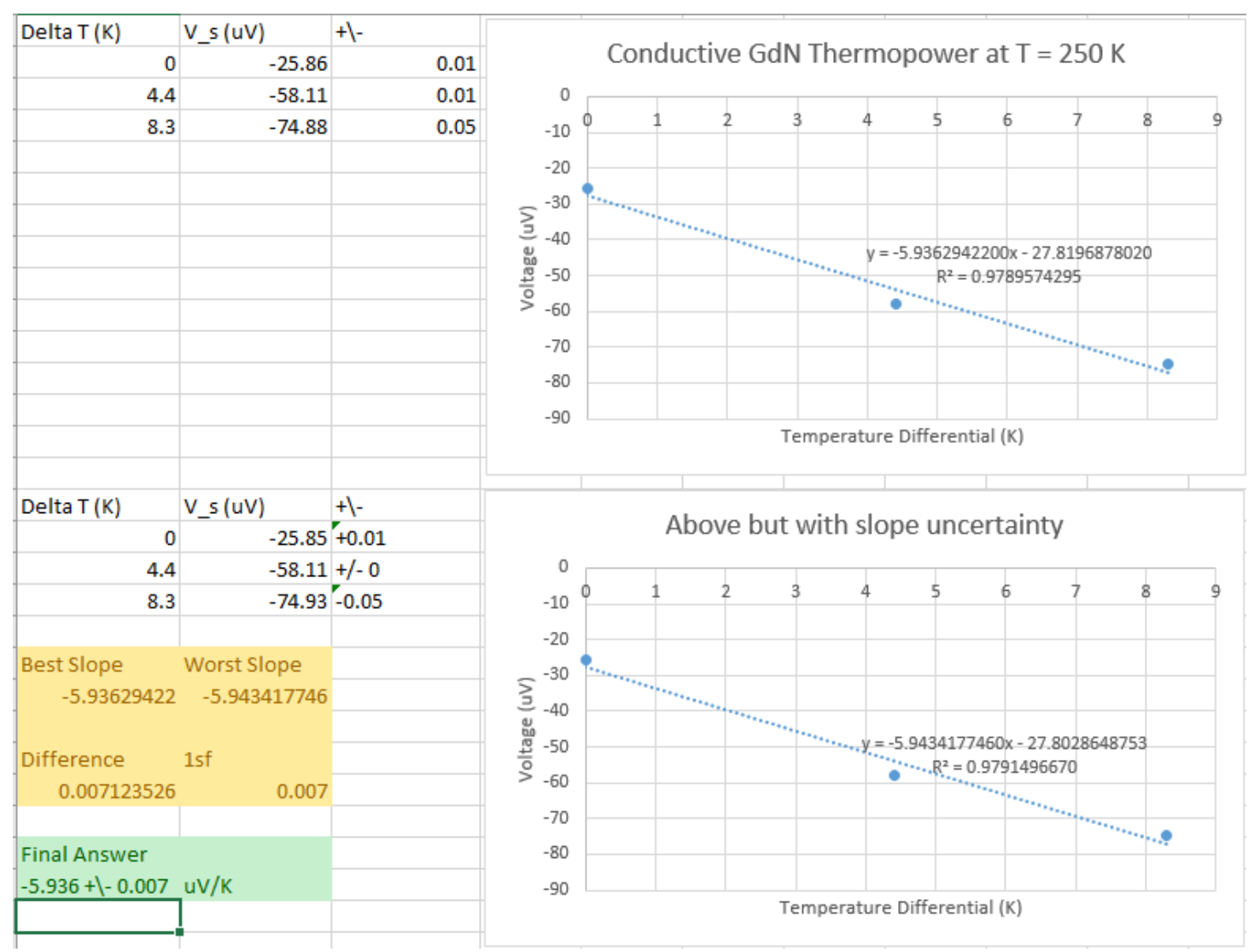

Figure A.8: Thermopower calculations at $\mathrm{T}=250 \mathrm{~K}$ 


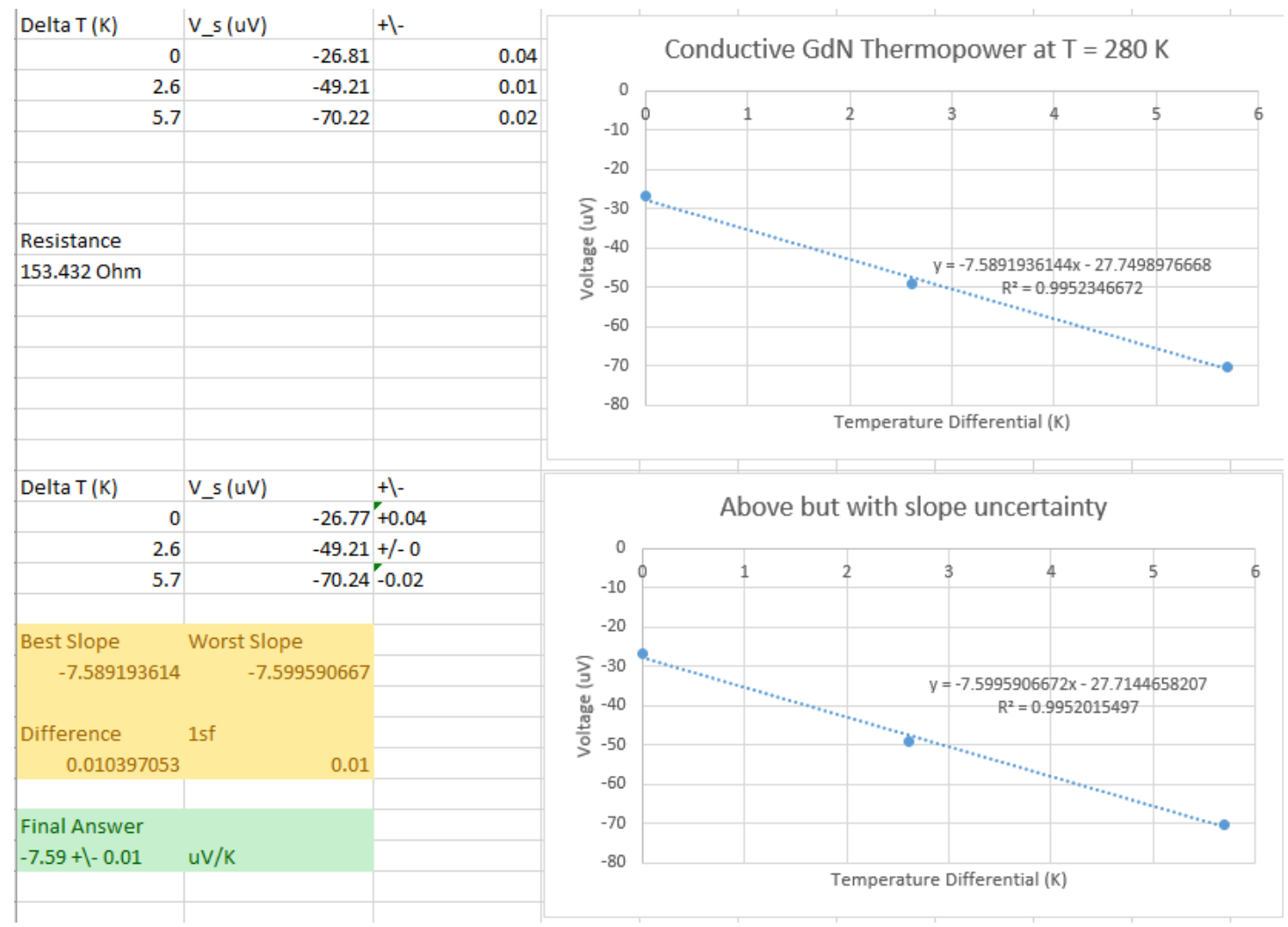

FiguRE A.9: Thermopower calculations at $\mathrm{T}=280 \mathrm{~K}$

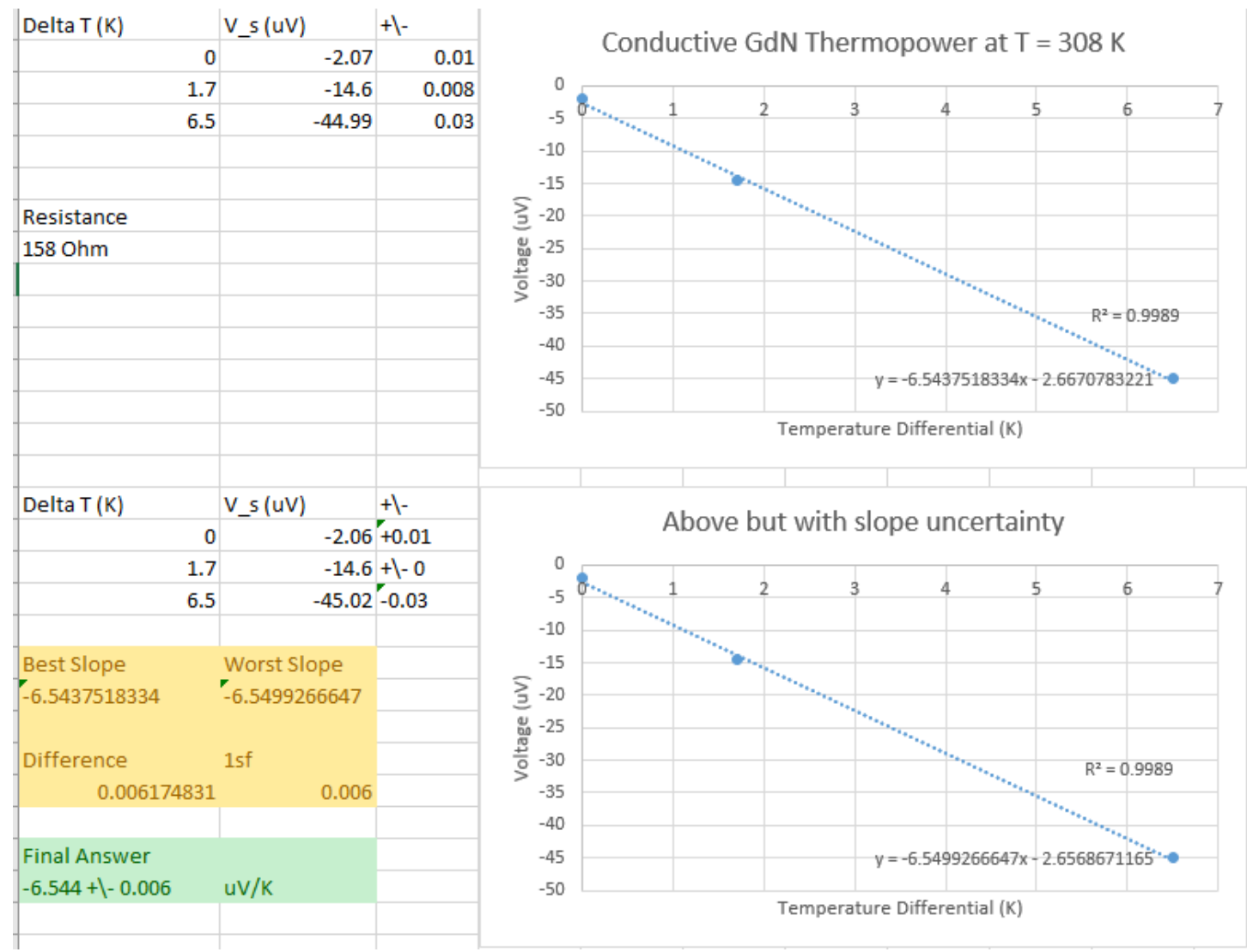

Figure A.10: Thermopower calculations at $\mathrm{T}=308 \mathrm{~K}$ 


\section{Appendix B}

\section{References}

1. Seebeck (1822). "Magnetische Polarisation der Metalle und Erze durch TemperaturDifferenz" [Magnetic polarization of metals and ores by temperature differences]. Abhandlungen der Kniglichen Akademie der Wissenschaften zu Berlin (in German): 265373.

2. Kittel, Charles (1953). Introduction to Solid State Physics. University of Michigan: Wiley

3. Leyland, W. J. H. (2008). "Energy Dependent Electron-Electron Scattering and and Spin Dynamics in a Two Dimensional Electron Gas."

Retrieved from https://arxiv.org/ftp/arxiv/papers/0803/0803.1230.pdf

4. Sakkopoulos, Sotirios Vitoratos, Evangelos Dalas, E Pandis, G Tsamouras, D. (1999). Thermopower sign reversal versus temperature and DC conductivity in polyaniline derivatives. Journal of Physics: Condensed Matter. 4. 2231. 10.1088/0953-8984/4/9/016.

5. Mott and Jones (1936) "The Theory of the Properties of Metals and Alloys" Oxford University Press

6. Landau, L. D.; Lifshitz, E. M. (1980). Statistical Physics Pt. 1. Course in Theoretical Physics. 5 (3rd ed.). Oxford: Pergamon Press. p. 193,196. ISBN 978-0-7506-3372-7.

7. Skinner, B. and Fu, L. (2018). Large, nonsaturating thermopower in a quantizing magnetic field.

Retrieved from http://arxiv-export-lb.library.cornell.edu/pdf/1706.06117 
8. Wang, H. H. (2018). Magnetic-field enhanced high-thermoelectric performance in topological Dirac semimetal $C d_{3} A s_{2}$.

Retrieved from https://arxiv.org/ftp/arxiv/papers/1802/1802.07868.pdf

9. Borca-Tasciuc, Theodorian Borca-Tasciuc, Diana-Andra Graham, Samuel Goods, Steve Kelly, J. Yang, Nancy. (2006). Annealing Effects on Mechanical and Transport Properties of Ni and Ni-Alloy Electrodeposits. Microelectromechanical Systems, Journal of. 15. 1051 - 1059. 10.1109/JMEMS.2006.879704.

10. K D D Rathnayaka 1985 J. Phys. E: Sci Instrum. 18380 